\title{
Fundamental properties of core-collapse supernova and GRB progenitors: predicting the look of massive stars before death ${ }^{\star}$
}

\author{
Jose H. Groh ${ }^{1}$, Georges Meynet ${ }^{1}$, Cyril Georgy ${ }^{2}$, and Sylvia Ekström ${ }^{1}$
}

\author{
1 Geneva Observatory, Geneva University, Chemin des Maillettes 51, 1290 Sauverny, Switzerland \\ e-mail: jose.groh@unige.ch \\ 2 Astrophysics group, EPSAM, Keele University, Lennard-Jones Labs, ST5 5BG Keele, UK
}

Received 16 May 2013 / Accepted 20 August 2013

\begin{abstract}
We investigate the fundamental properties of core-collapse supernova $(\mathrm{SN})$ progenitors from single stars at solar metallicity. For this purpose, we combine Geneva stellar evolutionary models with initial masses of $M_{\text {ini }}=20-120 M_{\odot}$ with atmospheric and wind models using the radiative transfer code CMFGEN. We provide synthetic photometry and high-resolution spectra of hot stars at the pre-SN stage. For models with $M_{\text {ini }}=9-20 M_{\odot}$, we supplement our analysis using publicly available MARCS model atmospheres of RSGs to estimate their synthetic photometry. We employ well-established observational criteria of spectroscopic classification and find that, depending on their initial mass and rotation, massive stars end their lives as red supergiants (RSG), yellow hypergiants (YHG), luminous blue variables (LBV), and Wolf-Rayet (WR) stars of the WN and WO spectral types. For rotating models, we obtained the following types of SN progenitors: WO1-3 $\left(M_{\text {ini }} \geq 32 M_{\odot}\right)$, WN10-11 $\left(25<M_{\text {ini }}<32 M_{\odot}\right)$, LBV $\left(20 \leq M_{\text {ini }} \leq 25 M_{\odot}\right)$, G1 Ia ${ }^{+}$ $\left(18<M_{\text {ini }}<20 M_{\odot}\right)$, and RSGs $\left(9 \leq M_{\text {ini }} \leq 18 M_{\odot}\right)$. For non-rotating models, we found spectral types WO1-3 $\left(M_{\text {ini }}>40 M_{\odot}\right)$, WN7-8 $\left(25<M_{\text {ini }} \leq 40 M_{\odot}\right)$, WN11h/LBV $\left(20<M_{\text {ini }} \leq 25 M_{\odot}\right)$, and RSGs $\left(9 \leq M_{\text {ini }} \leq 20 M_{\odot}\right)$. Our rotating models indicate that SN IIP progenitors are all RSG, SN IIL/b progenitors are 56\% LBVs and 44\% YHGs, SN Ib progenitors are 96\% WN10-11 and $4 \%$ WOs, and SN Ic progenitors are all WO stars. We find that the most massive and luminous SN progenitors are not necessarily the brightest ones in a given filter, since this depends on their luminosity, temperature, wind density, and the way the spectral energy distribution compares to a filter bandpass. We find that SN IIP progenitors (RSGs) are bright in the RIJHK filters and faint in the $U B$ filters. SN IIL/b progenitors (LBVs and YHGs), and SN Ib progenitors (WNs) are relatively bright in optical/infrared filters, while SN Ic progenitors (WOs) are faint in all optical filters. We argue that SN Ib and Ic progenitors from single stars should be undetectable in the available pre-explosion images with the current magnitude limits, in agreement with observational results.
\end{abstract}

Key words. stars: evolution - supernovae: general - stars: massive - stars: winds, outflows - gamma-ray burst: general stars: rotation

\section{Introduction}

Massive stars are ubiquitously present in the local and far Universe. Their short lives and the physics of star formation mean that they are easily outnumbered by their low-mass siblings like the Sun. Nevertheless, the impact of massive stars through cosmic time is far from negligible, since they are the main sources that input ionizing photons, energy, and recycled mass into the interstellar medium through stellar winds and supernova (SN) explosions.

Mass loss and angular momentum evolution play a key role in determining the properties of massive stars across their evolution and the properties of the SN progenitor and the ensuing explosion (for recent reviews see Maeder \& Meynet 2012; Langer 2012). The current generation of Geneva evolutionary models (Ekström et al. 2012; Georgy et al. 2012) predict that single rotating stars with initial masses $\left(M_{\text {ini }}\right)$ in the range $8 M_{\odot} \lesssim$ $M_{\text {ini }} \lesssim 17 M_{\odot}$ end their lives as red supergiant (RSG) stars before a SN event of the type IIP (i.e., with $\mathrm{H}$ lines dominating the spectrum and a plateau in the lightcurve). This scenario is supported well by the observations of SN IIP progenitors in

* New evolution models and model spectra are only available at the CDS via anonymous ftp to cdsarc.u-strasbg. fr (130.79.128.5) or via

http://cdsarc.u-strasbg.fr/viz-bin/qcat?]/A+A/558/A131 pre-explosion images, which have been shown to be RSGs with $8.5 M_{\odot} \lesssim M_{\text {ini }} \lesssim 16.5 M_{\odot}($ Smartt et al. 2009).

The agreement between theory and observations of SN progenitors is much less satisfactory for stars with $M_{\text {ini }} \gtrsim 17 M_{\odot}$. One problem is related to Galactic RSGs being observed to evolve from stars with $M_{\text {ini }}$ up to 25-30 $M_{\odot}$ (Levesque et al. 2005). If these stars die as RSGs, this raises the question of why no RSG more massive than about $16.5 M_{\odot}$ has been detected in pre-explosion images of SN progenitors ("the red supergiant problem", Smartt 2009). A possible solution to this problem would be the presence of circumstellar extinction around RSGs, which would underestimate the luminosity and mass determinations from the pre-explosion images (Smith et al. 2011a; Walmswell \& Eldridge 2012). It may also be that the most massive stars evolve away from the RSG phase and end their lifetime in a bluer portion of the Hertzsprung-Russell (HR) diagram (Vanbeveren et al. 1998; Salasnich et al. 1999; Yoon \& Cantiello 2010; Georgy et al. 2012; Georgy 2012; Meynet et al. 2013). For instance, rotating models predict that stars with $20 M_{\odot} \lesssim M_{\text {ini }} \lesssim 25 M_{\odot}$ are born as $\mathrm{O}$ dwarfs and exhibit a spectrum reminiscent of the rare luminous blue variable (LBV) stars before the SN explosion (Groh et al. 2013). The models indicate that a small amount of $\mathrm{H}$ is present in the envelope (a few $10^{-2} M_{\odot}$ ), making it difficult to infer the kind of SN that will follow core collapse (SN IIL, SN IIb, or even SN IIn if significant circumstellar material surrounds the progenitor). 
The situation becomes even hazier for stars with $M_{\text {ini }} \gtrsim$ $25 M_{\odot}$. According to evolutionary models, they reach the zeroage main sequence as O-type stars burning hydrogen in their cores and evolve to Wolf-Rayet (WR) type, helium-core burning stars (see e.g., Maeder \& Meynet 2000; Meynet \& Maeder 2003; Langer et al. 2007). Between the O-type and WR stages, the star may or may not go through an unstable, short-lived stage, usually associated to the LBV phase, and/or through a RSG phase. The models predict that core-collapse SN occur after the WR phase, and are of the type Ibc (Georgy et al. 2012, 2009). However, this scenario has yet to be observationally confirmed, specially because WR stars have not been directly detected as SN Ibc progenitors yet (Smartt 2009; Eldridge et al. 2013). The non-detection could be due to the intrinsic faintness of WRs in the optical bands, which are the ones usually available for the SN explosion site (Yoon et al. 2012), or because SN Ibc progenitors have lower masses and result from binary evolution (Podsiadlowski et al. 1992; Smartt 2009; Eldridge et al. 2013), or both. In addition, observations suggest that some massive stars can explode as $\mathrm{SNe}$ already during the LBV phase (e.g. Kotak \& Vink 2006; Smith et al. 2007; Pastorello et al. 2007; Gal-Yam \& Leonard 2009). Some of these progenitors likely had $M_{\text {ini }} \gtrsim 50 M_{\odot}$, which is much higher than the range of LBVs that can be SN progenitors based on single stellar evolution models (20 $M_{\odot} \lesssim M_{\text {ini }} \lesssim 25 M_{\odot}$; Groh et al. 2013).

Such glaring discrepancies between observations and the stellar evolution theory exposes a main gap in the understanding of massive stars and highlights our incomplete view of their post-main sequence evolution and fate. Several reasons exist for explaining our limit knowledge of massive stars. For instance, binarity plays an important role in the evolution of massive stars, as a significant fraction of massive stars seems to be in systems that will interact (Sana et al. 2012). Neglecting the effects of binaries on the properties of an average population of massive stars would likely yield inconsistent results. An equally important reason for our limited knowledge concerns the challenging comparison between observed data and stellar evolution models. Stellar evolution models are able to predict the stellar parameters only up to the stellar hydrostatic surface, which is not directly comparable to the observations when a dense stellar wind is present. This is the case for massive stars, in particular at their post-main sequence evolution, when they lose mass at enormous rates $\left(10^{-6}\right.$ to $\left.10^{-3} M_{\odot} \mathrm{yr}^{-1}\right)$. When the stellar wind becomes dense, eventually the photosphere becomes extended and is formed in a moving layer. Numerous emission lines arise in the wind, veiling (sometimes completely) the underlying spectrum from the hydrostatic surface that would be observable otherwise. As a consequence, the outputs of massive star evolution models, such as temperature, luminosity, and surface abundances at the hydrostatic surface, are difficult to be directly compared to observed quantities, such as a spectrum or a photometric measurement.

To solve this issue, it is necessary to couple stellar evolutionary calculations to radiative transfer models of the stellar atmosphere and wind. In Groh et al. (2013) we presented, for the first time, combined stellar evolution and atmospheric modeling at the pre-SN stage, for stars with $M_{\text {ini }}=20$ and $25 M_{\odot}$. Surprisingly, we found that these stars end their lives remarkably similar to LBVs, showing that in a single stellar evolution framework, massive stars can explode as LBVs.

Here we present a theoretical investigation of core-collapse SN progenitors from single stars with initial masses in the range $9-120 M_{\odot}$ at solar metallicity. We analyze how they appear to a distant observer, predicting observables such as the high-resolution spectrum, absolute magnitudes, colors, and bolometric corrections. The comparison between observations of SN progenitors and models can shed light on several properties of the progenitor, such as its mass, chemical composition, and initial rotation rate.

The motivations for our investigation are numerous. First, it allows a better constrain of the stellar evolution models. The classification of SNe generally traces the presence of chemical elements in the spectrum and the shape of the lightcurve (see e.g., Filippenko 1997 for a review). Studying the relative rate of the different core-collapse SN types is an important constraint for stellar evolution. This is because the chemical abundances in the ejecta supposedly reflects those from the progenitor before the SN explosion. Second, it allows a given observed SN rate to be linked to a given star formation history. This is possible only if we know sufficiently well the nature of the progenitors for the different types of core-collapse SNe. Finally, producing realistic observables from single-star evolution models also allows one to properly gauge whether a binary evolution scenario must necessarily be invoked to explain the observed properties of a given SN progenitor.

This paper is organized as follows. In Sect. 2 we describe our modeling approach. In Sect. 3 we revisit the expected fraction of the different kinds of core-collapse $\mathrm{SNe}$ from single stars and how they compare to recent observations. In Sect. 4 the location of the SN progenitors on the HR diagram, luminosity, and effective temperature are presented. Their spectroscopic appearance and spectral classification are performed in Sect. 5, while Sect. 6 compares our results with previous classifications of spectral types of SN progenitors based on chemical abundance criteria. The absolute magnitudes and bolometric corrections as a function of initial mass of the progenitor are presented in Sect. 7. We investigate the detectability of progenitors of the different types of core-collapse $\mathrm{SN}$ in Sect. 8. We discuss the surprising finding that WO stars are progenitor of type Ibc SNe in Sect. 9, while in Sect. 10 we discuss the possibility that LBVs are SN progenitors. We present our concluding remarks in Sect. 11.

In a series of forthcoming papers, we will present the results for the complete evolution of massive stars and for a larger metallicity range, and investigate the effects of several physical ingredients, such as magnetic fields, on the final appearance of massive stars.

\section{Physics of the models}

\subsection{Stellar evolution}

The evolutionary models are computed with the Geneva stellar evolution code. The majority of the models are those from Ekström et al. (2012). To better determine the mass ranges of the different SN progenitors, we compute new models with $M_{\text {ini }}$ of $16.5,18$, and $28 M_{\odot}$ (rotating models), and 23 and $50 M_{\odot}$ (nonrotating models $)^{1}$. Here we summarize the main characteristics of the code, and refer the reader to the aforementioned papers for further details. The models assume solar metallicity $(Z=0.014)$, initial abundances from Asplund et al. (2009), and the rotating models have initial rotational speed $\left(v_{\text {rot }}\right)$ of $40 \%$ of the critical velocity $\left(v_{\text {crit }}\right)$. The prescription for the rotational diffusion coefficients is taken from Zahn (1992) and Maeder (1997).

Mass loss is a key ingredient of the models, affecting not only the final position in the HR diagram but also the emerging spectrum of hot stars. Since the stellar evolution code requires

\footnotetext{
All models are publicly available through the webpage
} http: //obswww . unige. ch/Recherche/evol/-Database- 
previous knowledge of the spectral types to adopt a certain massloss recipe, criteria based on chemical abundances and effective temperatures are employed to estimate the type of massive star (OB, WR, RSG) at each time step (Smith \& Maeder 1991; Meynet \& Maeder 2003). The radiative mass-loss rates for OB stars follow the Vink et al. (2001) prescription, while for WR stars the Nugis \& Lamers (2000) and Gräfener \& Hamann (2008) prescriptions are employed. For the RSGs, when $\log \left(T_{\text {eff }} / \mathrm{K}\right)>3.7$, the de Jager et al. (1988) prescription is applied for initial masses of $15 M_{\odot}$ and above. For $\log \left(T_{\text {eff }} / \mathrm{K}\right) \leq$ 3.7, a linear fit to the data from Sylvester et al. (1998) and van Loon et al. (1999) is applied (see also Crowther 2001). For the RSGs in models below $15 M_{\odot}$, the Reimers $(1975,1977)$ relation (with $\eta=0.5$ ) is used.

Because of variations in the ionization level of hydrogen beneath the surface of the star during the RSG phase, significant changes in opacity may occur. Thus, some layers might exceed the Eddington limit, possibly driving instabilities. In this case, the radiative mass loss is increased by a factor of three in our models with initial mass above $18 M_{\odot}$, which matches the $\dot{M}$ determinations from van Loon et al. (2005). The effects of different $\dot{M}$ recipes during the RSG phase on the evolution of massive stars has been investigated by Georgy (2012), to where we refer the interested reader for further details.

As in Ekström et al. (2012), the stellar evolution models employed here terminate at the end of core-carbon burning. We do not expect significant variations in the surface properties after this phase. To verify this assumption, we computed the non-rotating $60 M_{\odot}$ model until core $\mathrm{O}$ burning, and negligible changes in $L_{\star}$ and $T_{\text {eff }}$ were seen $(\sim 0.01 \mathrm{dex})$.

\subsection{Atmospheric and wind modeling of hot stars}

The model spectra computed here are publicly available through a webpage ${ }^{2}$.

To compute the output spectra of stars with $T_{\star}>8000 \mathrm{~K}$ we used the atmospheric radiative transfer code CMFGEN (Hillier $\&$ Miller 1998). CMFGEN is a spherically-symmetric, fully line blanketed code that computes line and continuum formation in non-local thermodynamical equilibrium. Since all evolutionary models discussed here present negligible surface rotation at the pre-SN stage, the use of spherical symmetry is well justified. CMFGEN computes a self-consistent radiative transfer including the stellar hydrostatic surface and the wind. Wind (micro) clumping is included with a volume filling factor $(f)$ approach, which assumes dense clumps and a void interclump medium. The wind is also assumed to be unclumped close to the stellar surface and to acquire full clumpiness at large radii. All models computed here assume $f=0.1$. CMFGEN does not solve the momentum equation of the wind, and thus a hydrodynamical structure must be adopted. For the wind part, we assume a standard $\beta$-type law with $\beta=1$, while a hydrostatic solution is computed for the subsonic portion. This is applied up to 0.75 of the sonic speed, where the hydrostatic and wind solutions are merged. The wind terminal velocity $\left(v_{\infty}\right)$ is computed using the parametrization from Kudritzki \& Puls (2000) for OB stars and LBVs, and from Nugis \& Lamers (2000) for WR stars of the WN and WC type. For WO stars, an iterative scheme is adopted. We initially compute a spectrum with the value of $v_{\infty}$ as given by the Nugis \& Lamers (2000) recipe, which is typically at most $\sim 2800 \mathrm{~km} \mathrm{~s}^{-1}$. If a WO-type spectrum arises, we recompute a spectrum with $v_{\infty}=5000 \mathrm{~km} \mathrm{~s}^{-1}$ which is more representative

\footnotetext{
2 http://obswww. unige.ch/Recherche/evol/-Database-
}

of the observed Galactic WO stars (Drew et al. 2004; Sander et al. 2012).

We use the outputs from the stellar structure calculations with the Geneva code, such as the radius, luminosity, mass, and surface abundances, as inputs in CMFGEN. For consistency, we adopt the same mass-loss rate recipe as that used by the Geneva evolution code. We use the temperature structure of the stellar envelope to merge the CMFGEN solution and the stellar structure solution. The outputs from the CMFGEN calculations that we discuss here are the synthetic spectrum, photometry, and the effective temperature $T_{\text {eff }}$, defined as the temperature of the layer where the Rosseland optical depth is $2 / 3$. The values of $T_{\star}$ quoted here correspond to those predicted by the Geneva stellar evolution code without the correction due to the optical depth of the wind, and not to the temperature at a fixed Rosseland optical depth (usually 20).

\subsection{Atmospheric modeling of cool stars}

A realistic atmospheric analysis of luminous cool stars requires the inclusion of convection and $\mathrm{H}^{-}$and molecular opacities, which at the moment are not included in CMFGEN. Here, we employ the publicly available MARCS models (Gustafsson et al. 2008) to perform synthetic photometry of the SN progenitors that have $T_{\text {eff }}=3400-5400 \mathrm{~K}$ and thus are RSGs or YHGs. We use the model grids that have abundances corresponding to $\mathrm{CN}$-processed material, which is characteristic of RSGs at the pre-SN stage. A mass of $5 M_{\odot}$ and turbulent velocity of $2 \mathrm{~km} \mathrm{~s}^{-1}$ are assumed. Since these MARCS models are available only at coarse $T_{\text {eff }}$ sampling (3400, 3600, and $3800 \mathrm{~K}$ for RSGs; 5000, $5250,5500,5750 \mathrm{~K}$ for YSG/YHG) and not at the exact luminosities predicted by the evolutionary models, the magnitudes and bolometric corrections of the cool SN progenitors were estimated by:

1) linearly interpolating the magnitudes (bolometric corrections) of two bracketing MARCS models in $\log T_{\text {eff }}$ space to the desired $T_{\text {eff }}$ value predicted by the Geneva code. The bracketing MARCS models were previously scaled to the same luminosity;

2) scaling the interpolated magnitudes to the luminosities predicted by the Geneva code.

This is a zeroth-order approximation to estimate the magnitudes and should be checked in the future against MARCS models computed specifically for the physical parameters $\left(L_{\star}\right.$, $T_{\text {eff }}, M_{\star}$, abundances) found at the pre-SN stage. Since these are unavailable at the moment, synthetic spectra of RSGs and YHGs are not provided. Still, the magnitude estimates computed here provide important insights into the nature of core-collapse SN progenitors.

\section{The core-collapse SN fraction from single stars}

Before computing the stellar spectra and colors associated to the various SN progenitors predicted by the grids of models of Ekström et al. (2012), it is interesting to see whether these models can reproduce or not the observed rates of various types of core-collapse SNe. This has already been discussed extensively in Georgy et al. (2012), which determined the relative rates of the different core-collapse SN types and compared to the observational sample from Boissier \& Prantzos (2009). Here we revisit this topic, given that two relatively large samples of observational core-collapse SN data have 
Table 1. Core-collapse SN fractions from our models compared to observed samples.

\begin{tabular}{lcccc}
\hline \hline SN Type & $\begin{array}{c}\text { Non-rot. model } \\
(\%)\end{array}$ & $\begin{array}{c}\text { Rot. model } \\
(\%)\end{array}$ & $\begin{array}{c}\text { Obs. E13 } \\
(\%)\end{array}$ & $\begin{array}{c}\text { Obs. S11 } \\
(\%)\end{array}$ \\
\hline IIP & 70.7 & 64.9 & $55.5 \pm 6.6$ & $48.2_{-5.6}^{+5.7}$ \\
IIL + IIb & 16.2 & 13.1 & $15.1 \pm 3.4$ & $17.0_{-4.0}^{+4.6}$ \\
IIn & - & - & $2.4 \pm 1.4$ & $8.8_{-2.9}^{+3.3}$ \\
IIpec & - & - & $1.0 \pm 0.9$ & - \\
Ib & 8.1 & 7.8 & $9.0 \pm 2.7$ & $8.4_{-2.6}^{+3.1}$ \\
Ic & 5.0 & 14.2 & $17.0 \pm 3.7$ & $17.6_{-3.8}^{+4.2}$ \\
\hline
\end{tabular}

been recently released (Smith et al. 2011a, hereafter S11, and Eldridge et al. 2013, hereafter E13). We assume, as in Georgy et al. (2012), a Salpeter-type initial mass function, that the lower and upper initial mass limit for core-collapse SNe are 8 and $120 M_{\odot}$, respectively, that $\mathrm{SNe}$ occur even when black holes are formed, and that all SNe are observable (see also Heger et al. 2003). For the SN classification we assume, as in Georgy et al. (2012), the following chemical abundances in the ejecta:

$$
\begin{aligned}
& \text { - a SN IIP has more than } 2 M_{\odot} \text { of } \mathrm{H} \text {; } \\
& \text { - a SN IIL/b has between } 0 \text { and } 2 M_{\odot} \text { of H; } \\
& \text { - a SN Ib has no H and more than } 0.6 M_{\odot} \text { of He; } \\
& \text { - a SN Ic has no H and less than } 0.6 M_{\odot} \text { of He. }
\end{aligned}
$$

We present in Table 1 the fraction of the different SN types predicted by our non-rotating and rotating models (see also Georgy et al. 2012), and those of the S11 and E13 observational samples. A detailed comparison between both observational samples has been performed by E13.

As one can see, the $\mathrm{SN}$ rates yielded by our rotating models are mostly within the errors of the S11 and E13 observations, with the exception of the SN IIP fraction, which is $1.5 \sigma(2.9 \sigma)$ above the E13 (S11) values. For the non-rotating models, the SN IIP relative rate is too high compared to the observed one, while the SN Ic relative rate is too low. We stress that the $\mathrm{SN}$ rates are quite sensitive to metallicity (e.g., Georgy et al. 2009) and, while our models discussed here are at solar metallicity, the observed samples likely include a range of metallicities around solar.

Interesting remarks can be deduced from these results. First, from a pure theoretical point of view, the different results obtained with and without rotation illustrate the sensitivity of the predicted supernova rate on axial rotation. Second, for a proper comparison with the observations, population synthesis models are needed, taking a range of initial rotational velocities into account (our rotating models have initial $v_{\text {rot }} / v_{\text {crit }}=0.4$ ). Third, the current rotating models are in good shape for reproducing the observed rates, while non-rotating models are in a less good position. This is in line with other evidences for some rotational mixing being active in massive stars (see e.g. the review by Maeder \& Meynet 2012 and references therein). Fourth, the above comparisons somewhat support the current rotating models. However, it is difficult to draw very firm conclusions here since we cannot discard the possibility that some of our models would produce some faint or even failed SN event. In that sense, the theoretical SN Ibc rates are likely overestimated. If this turns out to occur, it may indicate that other channels are important for explaining the observed rates, as for instance the close binary evolution channel (S11, E13).

Since a significant fraction of massive stars are in binary systems (e.g. Sana et al. 2012), it would not be surprising if a sizable fraction of core-collapse SN progenitors evolve under the influence of a close companion, for instance via tides and mass transfer (de Mink et al. 2013). The modeling of SN Ibc lightcurves also provides strong constraints to the amount of ejecta mass (e.g. Drout et al. 2011; Dessart et al. 2011; Cano 2013), with an average value of $4.6 M_{\odot}$ (Cano 2013). Since our models predict SN Ibc ejecta masses in the range 6.7-21.8 $M_{\odot}$ (Georgy et al. 2012), it is thought that part of the SN Ibc progenitors could come from binary evolution. Based on the core-collapse SN rates, both $\mathrm{S} 11$ and E13 independently find that roughly $50 \%$ of the progenitors follow single star evolution and 50\% evolve in interacting binary systems.

Ultimately, the best way to distinguish between SN progenitors that come from single or binary evolution is the direct detection of the progenitor in pre-explosion images. To fully grasp all the information from these observations, a comparison with stellar evolution models is needed. The main driver of this paper is thus to produce observables out of single massive star evolution models at the pre-SN stage that can be directly compared to observations.

\section{Location of SN progenitors in the HR diagram: luminosity and effective temperature}

Figure 1 shows an HR diagram with evolutionary tracks of rotating and non-rotating stars obtained with the Geneva stellar evolution code (Ekström et al. 2012; Georgy et al. 2012), as well as the additional models computed for the purpose of this work, with initial mass in the range 9 to $120 M_{\odot}$ at solar metallicity. Here we focus on the pre-SN stage (diamonds and squares in Fig. 1).

The temperatures of the evolutionary tracks shown in Fig. 1 refer to those computed by the Geneva code without the correction due to optical-depth effects of the stellar wind. To obtain the effective temperature, optical depth effects due to the presence of an atmosphere and dense stellar wind have to be taken into account, and an atmospheric modeling must be performed. We indicate in Fig. 1 the values of $T_{\text {eff }}$ obtained with CMFGEN for hot stars (squares). These values thus correspond to the $T_{\text {eff }}$ that one should employ when comparing with the observed $T_{\text {eff }}$. For RSGs, we assume that the presence of a wind does not affect the determination of $T_{\text {eff }}$, thus $T_{\text {eff }}=T_{\star}$. The other fundamental parameters of the $\mathrm{SN}$ progenitors at solar metallicity are summarized in Table 2.

Rotating models with $\sim 9 M_{\odot} \leq M_{\text {ini }} \leq 16.5 M_{\odot}\left(\sim 9 M_{\odot} \leq\right.$ $M_{\text {ini }} \leq 23 M_{\odot}$ for non rotating ones) are RSGs at the preSN stage (Ekström et al. 2012). They end their lives with $T_{\text {eff }}=3480-4410 \mathrm{~K}$. The rotating $18 M_{\odot}$ model is a luminous yellow star at the pre-SN stage, with $T_{\mathrm{eff}}=5382 \mathrm{~K}$ and $L_{\star}=1.5 \times 10^{5} L_{\odot}$.

The rotating models with $M_{\text {ini }}=20,25$, and $28 M_{\odot}$ evolve back to the blue after the RSG phase, and develop strong winds as they evolve towards the end stage. The $20 M_{\odot}$ model achieves $L_{\star}=1.9 \times 10^{5} L_{\odot}$ and $T_{\text {eff }} \simeq 19540 \mathrm{~K}$, while the $25 M_{\odot}$ model has $L_{\star}=3.2 \times 10^{5} L_{\odot}$ and $T_{\text {eff }} \simeq 20000 \mathrm{~K}$ at the end of its evolution (Groh et al. 2013). The rotating $28 M_{\odot}$ model reaches $L_{\star}=3.5 \times 10^{5} L_{\odot}$ and $T_{\text {eff }} \simeq 26800 \mathrm{~K}$ at the pre-SN stage. In the non-rotating case, the $M_{\text {ini }}=25 M_{\odot}$ model ends its life with $T_{\text {eff }} \simeq 26300 \mathrm{~K}$ and $L_{\star}=2.4 \times 10^{5} L_{\odot}$. We note that these models are close to the bistability limit of line-driven-winds, when abrupt changes in mass loss occur (Vink et al. 1999). In some cases, the models flirt with this limit close to the end stages and oscillates from one side of the bistability limit to the other, 
Jose H. Groh et al.: Fundamental properties of core-collapse SN progenitors
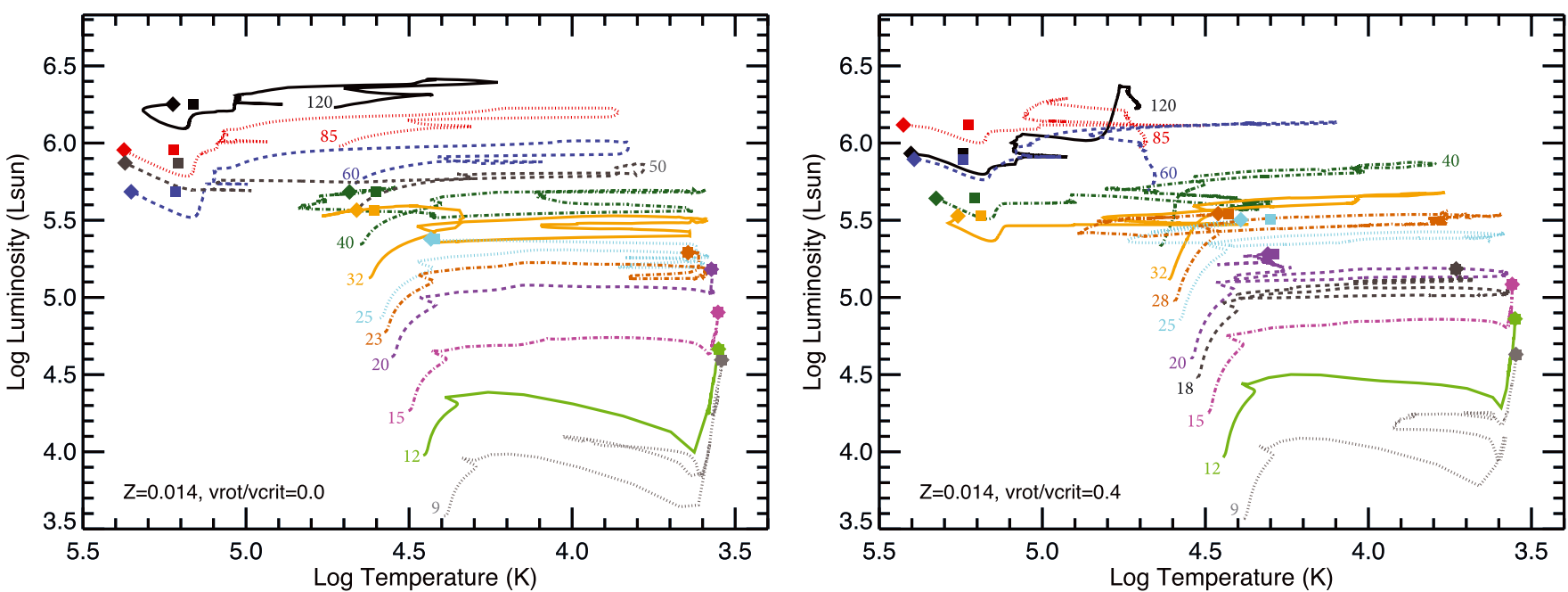

Fig. 1. Left: HR diagram showing evolutionary tracks of non-rotating stars with initial masses between $9 M_{\odot}$ and $120 M_{\odot}$ at solar metallicity $(Z=0.014)$. The diamonds correspond to the temperature computed by the Geneva code and are not corrected by the effects of the stellar wind $\left(T_{\star}\right)$, and the squares correspond to the values of $T_{\text {eff }}$ computed either with CMFGEN (for $T_{\text {eff }}>8000 \mathrm{~K}$ ), or assumed to be equal to $T_{\star}$ for RSGs (causing the squares and diamonds to overlap). Right: similar to the left panel, but for rotating stars with initial $v_{\text {rot }} / v_{\text {crit }}=0.4$. Except for the non-rotating 23 and $50 M_{\odot}$ and the rotating 18 and $28 M_{\odot}$ models, evolutionary tracks are from Ekström et al. (2012).

Table 2. Properties of the core-collapse SN progenitor models at solar metallicity.

\begin{tabular}{|c|c|c|c|c|c|c|c|c|c|c|c|c|}
\hline $\begin{array}{l}M_{\mathrm{ini}} \\
\left(M_{\odot}\right) \\
\end{array}$ & $\begin{array}{c}M_{\text {prog. }}{ }^{a} \\
\left(M_{\odot}\right) \\
\end{array}$ & $\begin{array}{l}\mathrm{Age}^{a} \\
\text { (Myr) }\end{array}$ & $\begin{array}{l}L_{\star}{ }^{a} \\
\left(L_{\odot}\right)\end{array}$ & $\begin{array}{r}T_{\star}^{a, b} \\
(\mathrm{~K}) \\
\end{array}$ & $\begin{array}{l}T_{\text {eff }} \\
(\mathrm{K})\end{array}$ & $\begin{array}{c}R_{\star} \\
\left(R_{\odot}\right) \\
\end{array}$ & $\begin{array}{l}R_{\text {phot }} \\
\left(R_{\odot}\right)\end{array}$ & $\begin{array}{c}\dot{M}^{a} \\
\left(M_{\odot} \mathrm{yr}^{-1}\right) \\
\end{array}$ & $\begin{array}{c}v_{\infty} \\
\left(\mathrm{km} \mathrm{s}^{-1}\right)\end{array}$ & $\beta$ & Sp. Type & SN Type \\
\hline \multicolumn{13}{|c|}{ non-rotating models } \\
\hline 9 & 8.8 & 30.3 & 39401 & 3480 & 3480 & 547.49 & 547.49 & $9.7 \times 10^{-7}$ & - & - & $K-M I$ & IIP \\
\hline 12 & 11.3 & 17.8 & 46259 & 3556 & 3556 & 567.94 & 567.94 & $1.3 \times 10^{-6}$ & - & - & $K-M I$ & IIP \\
\hline 15 & 13.25 & 12.5 & 79999 & 3571 & 3571 & 740.91 & 740.91 & $3.2 \times 10^{-6}$ & - & - & $K-M I$ & IIP \\
\hline 20 & 8.8 & 8.7 & 152291 & 3741 & 3741 & 931.42 & 931.42 & $2.0 \times 10^{-5}$ & - & - & $K-M I$ & IIL/b \\
\hline 23 & 7.6 & 7.6 & 195030 & 4410 & 4410 & 758.29 & 758.29 & $4.4 \times 10^{-5}$ & - & - & $K-M I$ & IIL/b \\
\hline 25 & 8.2 & 7.09 & $2.4 \mathrm{E}+05$ & 27116 & 26330 & 22.20 & 23.49 & $9.7 \times 10^{-6}$ & 694 & 1.0 & WN11h/LBV & IIL/b \\
\hline 32 & 10.9 & 5.81 & $3.7 \mathrm{E}+05$ & 45790 & 40480 & 9.65 & 12.32 & $2.9 \times 10^{-5}$ & 1200 & 1.0 & WN7-8o & $\mathrm{Ib}$ \\
\hline 40 & 12.7 & 4.97 & $4.8 \mathrm{E}+05$ & 48183 & 40006 & 9.99 & 14.4 & $4.2 \times 10^{-5}$ & 1291 & 1.0 & WN7-8o & $\mathrm{Ib}$ \\
\hline 50 & 16.53 & 4.34 & $7.4 \mathrm{E}+05$ & 235060 & 161200 & 0.52 & 1.10 & $1.2 \times 10^{-5}$ & 5000 & 1.0 & WO1-3 & $\mathrm{Ib}$ \\
\hline 60 & 12.4 & 3.97 & $4.8 \mathrm{E}+05$ & 225077 & 164600 & 0.46 & 0.85 & $1.5 \times 10^{-5}$ & 5000 & 1.0 & WO1-3 & Ic \\
\hline 85 & 18.5 & 3.40 & $9.0 \mathrm{E}+05$ & 236900 & 166900 & 0.56 & 1.13 & $2.2 \times 10^{-5}$ & 5000 & 1.0 & WO1-3 & Ic \\
\hline 120 & 30.7 & 3.00 & $1.8 \mathrm{E}+06$ & 167700 & 145100 & 1.59 & 2.11 & $3.1 \times 10^{-5}$ & 5000 & 1.0 & WO1-3 & $\mathrm{Ib}$ \\
\hline \multicolumn{13}{|c|}{ rotating models, initial $v_{\text {rot }} / v_{\text {crit }}=0.4$} \\
\hline 9 & 8.5 & 35.5 & 42802 & 3528 & 3528 & 555.08 & 555.08 & $1.1 \times 10^{-6}$ & - & - & $K-M I$ & IIP \\
\hline 12 & 10.22 & 20.7 & 72652 & 3550 & 3550 & 714.24 & 714.24 & $2.7 \times 10^{-6}$ & - & - & $K-M I$ & IIP \\
\hline 15 & 11.07 & 15.0 & 121442 & 3623 & 3623 & 886.73 & 886.73 & $6.5 \times 10^{-6}$ & - & - & $K-M I$ & IIP \\
\hline 16.5 & 12.14 & 13.0 & 126610 & 3645 & 3645 & 894.92 & 894.92 & $7.0 \times 10^{-6}$ & - & - & $K-M I$ & IIP \\
\hline 18 & 6.32 & 11.7 & 153052 & 5382 & 5382 & 451.20 & 451.20 & $1.7 \times 10^{-5}$ & - & - & $G 1 I a^{+}$ & IIL/b \\
\hline 20 & 7.1 & 10.4 & $1.9 \mathrm{E}+05$ & 20355 & 19540 & 35.30 & 38.11 & $1.2 \times 10^{-5}$ & 272 & 1.0 & LBV & $\mathrm{IIL} / \mathrm{b}$ \\
\hline 25 & 9.6 & 8.60 & $3.2 \mathrm{E}+05$ & 24625 & 20000 & 31.1 & 46.96 & $4.6 \times 10^{-5}$ & 326 & 1.0 & LBV & $\mathrm{Ib}$ \\
\hline 28 & 10.8 & 7.92 & $3.5 \mathrm{E}+05$ & 28882 & 26823 & 23.68 & 26.26 & $2.6 \times 10^{-5}$ & 415 & 1.0 & WN10-11 & $\mathrm{Ib}$ \\
\hline 32 & 10.1 & 7.22 & $3.4 \mathrm{E}+05$ & 181500 & 154100 & 0.58 & 0.81 & $1.2 \times 10^{-5}$ & 5000 & 1.0 & WO1 & Ic \\
\hline 40 & 12.2 & 6.17 & $4.4 \mathrm{E}+05$ & 211700 & 161100 & 0.49 & 0.85 & $1.4 \times 10^{-5}$ & 5000 & 1.0 & WO1-2 & Ic \\
\hline 60 & 18.9 & 4.86 & $8.6 \mathrm{E}+05$ & 247100 & 174500 & 0.48 & 1.01 & $2.0 \times 10^{-5}$ & 5000 & 1.0 & WO1-3 & Ic \\
\hline 85 & 26.2 & 4.06 & $1.3 \mathrm{E}+06$ & 266700 & 168400 & 0.54 & 1.34 & $2.8 \times 10^{-5}$ & 5000 & 1.0 & WO1-3 & $\mathrm{Ib}$ \\
\hline 120 & 18.9 & 3.55 & $8.6 \mathrm{E}+05$ & 252800 & 174500 & 0.48 & 1.01 & $2.0 \times 10^{-5}$ & 5000 & 1.0 & WO1 & Ic \\
\hline
\end{tabular}

Notes. From left to right, the columns correspond to the initial mass on the main sequence $\left(M_{\text {ini }}\right)$, the SN progenitor mass $\left(M_{\text {prog. }}\right)$, and the age, bolometric luminosity $\left(L_{\star}\right)$, temperature at the hydrostatic surface $\left(T_{\star}\right)$, effective temperature $\left(T_{\text {eff }}\right)$, radius of the hydrostatic surface $\left(R_{\star}\right)$, photospheric radius $\left(R_{\text {phot }}\right)$, mass-loss rate $(\dot{M})$, wind terminal velocity $\left(v_{\infty}\right)$, steepness of the velocity law $(\beta)$, and spectral type at the pre-SN stage. The last column corresponds to the SN type as computed by Georgy et al. (2012). Spectral types in italic were not obtained via classification of the spectra, and thus are tentative. ${ }^{(a)}$ From Ekström et al. (2012), except for the non-rotating 23 and $50 M_{\odot}$ and the rotating 18 and $28 M_{\odot}$ models, which were computed for this work. ${ }^{(b)}$ This is defined here as the temperature computed by the Geneva code without the correction due to optical-depth effects of the stellar wind. 
having an erratic behavior in $\dot{M}$. Therefore, the final position of these models in the HR diagram are probably relatively coarse within a couple thousand $\mathrm{K}$.

For stars with $M_{\text {ini }}=32 M_{\odot}$ to $40 M_{\odot}$, there are huge differences in the $T_{\text {eff }}$ of rotating and non-rotating models at the pre-SN stage. This happens because rotation increases mixing, which brings He burning processed material $(\mathrm{C}$ and $\mathrm{O})$ closer to the surface compared to when rotation is absent. As the star evolves, mass loss removes the outer layers and expose $\mathrm{C}$ and $\mathrm{O}$ at the stellar surface. Therefore, while the $M_{\mathrm{ini}}=32 M_{\odot}$ and $40 M_{\odot}$ non-rotating models have $T_{\mathrm{eff}} \simeq 40000 \mathrm{~K}$, the rotating ones reach $T_{\text {eff }} \simeq 150000-160000 \mathrm{~K}$ at the pre-SN stage. The values of $L_{\star}$ of both rotating and non-rotating models are comparable, with the non-rotating models having a slightly higher luminosity.

We obtain that stars with $M_{\text {ini }}=50 M_{\odot}$ to $120 M_{\odot}$, both in rotating and non-rotating models, end their lives with extremely high values of $T_{\text {eff }} \simeq 145000-175000 \mathrm{~K}$. We notice that the rotating models have a tendency to reach the pre-SN stage with higher $T_{\text {eff }}$ than the non-rotating models. The difference is about $5000-10000 \mathrm{~K}$ at $60 M_{\odot}$ and $85 M_{\odot}$, but rises considerably to $30000 \mathrm{~K}$ at $120 M_{\odot}$. This occurs because the rotating models have higher $T_{\star}$ than non-rotating models. As discussed by Georgy et al. (2012), the $60 M_{\odot}$ and $85 M_{\odot}$ rotating models finish with a higher luminosity ( 0.2 and 0.15 dex, respectively) than the corresponding non-rotating models, while the rotating $120 M_{\odot}$ model finishes at a much lower luminosity (by 0.3 dex) than the non-rotating model.

\section{Spectra and spectral type classification of SN progenitors}

While the spectral types of low-mass stars can be securely obtained from $T_{\text {eff }}, \log g$, and $L_{\star}$ only, the same is not true for massive stars, in particular at the end of their evolution. This is because massive stars are characterized by dense outflows, which have an impact in the spectral morphology. In addition to the luminosity, temperature, effective gravity, and abundances, several other quantities may affect the emerging spectrum. Among these, the mass-loss rate and wind terminal velocity are the ones that influence most the appearance of SN progenitors. Here, we compute the synthetic spectra of SN progenitors with CMFGEN, based on the output from stellar evolution models. Therefore, it is not entirely surprising that the spectral types that we derive in this section are different from those widely quoted in the literature which, in the absence of a spectrum, were estimated using chemical abundance criteria.

Figures 2 and 3 show the synthetic optical spectra of SN progenitors with $M_{\text {ini }}=20 M_{\odot}$ to $120 M_{\odot}$. We display representative spectral regions that allow a broad illustration of the spectral morphology and spectral type determination. The ultraviolet to infrared spectra are available in the online version. To classify the synthetic spectra, we used the criteria from Crowther et al. (1998) for WO and WC stars, and Smith et al. (1996) and Crowther et al. (1995) for WN stars. Models that have spectrum similar to observed bona-fide LBVs, such as AG Car, P Cygni, and HR Car, have their spectral type listed as LBVs. While we recognize that formally there is no "LBV" spectral classification, we opted to use this classification since there is no objective spectral classification criteria of stars with dense winds that have $8000 \mathrm{~K} \lesssim T_{\text {eff }} \lesssim 25000 \mathrm{~K}$. The spectra of these stars have been commonly referred to in the literature as "P Cygni-type", "iron", and "slash" stars (see, e.g., Walborn \& Fitzpatrick 2000; Clark et al. 2012a). The results of our spectral classification of the SN progenitors are summarized in the last column of Table 2.

The pre-SN optical spectrum of the non-rotating model with $M_{\text {ini }}=25 M_{\odot}$ is displayed in Fig. 2a. The spectrum is characterized by strong emission of H I lines with shallow P-Cyg absorption profiles. Moderate emission of He I, N II, and Si IV lines are also seen, while N III lines are weak or absent. Following the criteria from Crowther et al. (1995), the presence of $\mathrm{H}$, the N II lines being stronger than those of N III, and the presence of He II $\lambda 4686$ indicate a WN11h classification. Indeed, the non-rotating $25 M_{\odot}$ model spectrum is similar to that of the LBV AG Carinae at visual minimum (1989 June), when it had a WN11h spectral type (Smith et al. 1994; Leitherer et al. 1994; Stahl et al. 2001; Groh et al. 2009b). The main difference is that the model spectrum has broader lines, since its $v_{\infty}=694 \mathrm{~km} \mathrm{~s}^{-1}$ is about twice that of AG Car in 1989 June $\left(300 \mathrm{~km} \mathrm{~s}^{-1}\right.$, Groh et al. 2009b). Another marked difference is the ratio between $\mathrm{H}$ and $\mathrm{He} \mathrm{I}$ lines, which is smaller in the model spectrum compared to the observations of AG Car. This is due to a combination of higher He abundance and higher $T_{\text {eff }}$ in the model spectrum compared to those of AG Car in 1989 June. We find that the non-rotating $25 M_{\odot}$ model has a peculiar appearance, looking something in between an LBV and WN11h star, and classify it as WN11h/LBV. Nevertheless, we note that all known WN11h stars are LBVs (or candidates) at their visual minimum, such as AG Car, Hen 3-519, and HDE 316285, so the spectral type WN11h seems to be exclusively linked to LBVs.

The synthetic spectra of the non-rotating models with $32 M_{\odot}<M_{\text {ini }}<40 M_{\odot}$ are shown in Fig. 2b. Our models indicate that the non-rotating $32 M_{\odot}$ and $40 M_{\odot}$ models have at their final stage an optical spectrum that shows strong He II $\lambda$ 44686, N III $\lambda$ 44640, and N IV $\lambda$ 44057. Numerous He I lines are also present with strong P-Cygni profile, such as He I $\lambda 5876$ and He I 26678 . These features are characteristic of a WN star of late spectral type (WNL). The He ionization structure indicates a WN 7 spectral type, but close to the transition to WN 8. In our models, the continuum-to-peak ratio of $\mathrm{He}$ I $\lambda 5411 / \mathrm{He}$ I $\lambda 5876 \simeq$ 0.71 , while the boundary between WN7 and WN8 spectral types lies at He I $\lambda 5411 / \mathrm{He}$ I $\lambda 5876=0.65$ (Smith et al. 1996). The $\mathrm{N}$ ionization structure is broadly supportive of a WN7-8 classification. Our synthetic spectra have line widths not extremely broad, and are indicative of a WN7-8o subtype. For comparison, we present the observed spectrum of a typical Galactic WN7 (WR120) and WN8 star (WR 123), obtained from the atlas of Hamann et al. (1995).

Figure 2c displays the SN progenitor spectra from nonrotating models with $50 M_{\odot}<M_{\text {ini }}<120 M_{\odot}$. Their optical spectra are characterized by broad emission lines of O VI $\lambda 3811$ and C IV 15808 . This implies that they have WO spectral types at the pre-SN stage, confirming previous indications that even at solar metallicity, massive stars can have their final stage as WO stars (Sander et al. 2012; Yoon et al. 2012). Here, we proceed one step further by computing the synthetic spectra, which allows us to derive spectral types and estimate the mass range where $\mathrm{WO}$ are $\mathrm{SN}$ progenitors. We obtain spectral types WO 1-3, depending on whether primary (ratio of EW of O VI $\lambda 3818 / \mathrm{OV} \lambda 5590)$ or secondary classification criteria (O VI $\lambda 3818 / \mathrm{C}$ IV $\lambda$ 15808) are employed (see Crowther et al. 1998). Nevertheless, it is clear that the SN progenitors with $50 M_{\odot}<M_{\text {ini }}<120 M_{\odot}$ should be the hottest massive stars known.

We note that the synthetic spectrum of the non-rotating $120 M_{\odot}$ model has emission line strengths comparable to those 
Jose H. Groh et al.: Fundamental properties of core-collapse SN progenitors
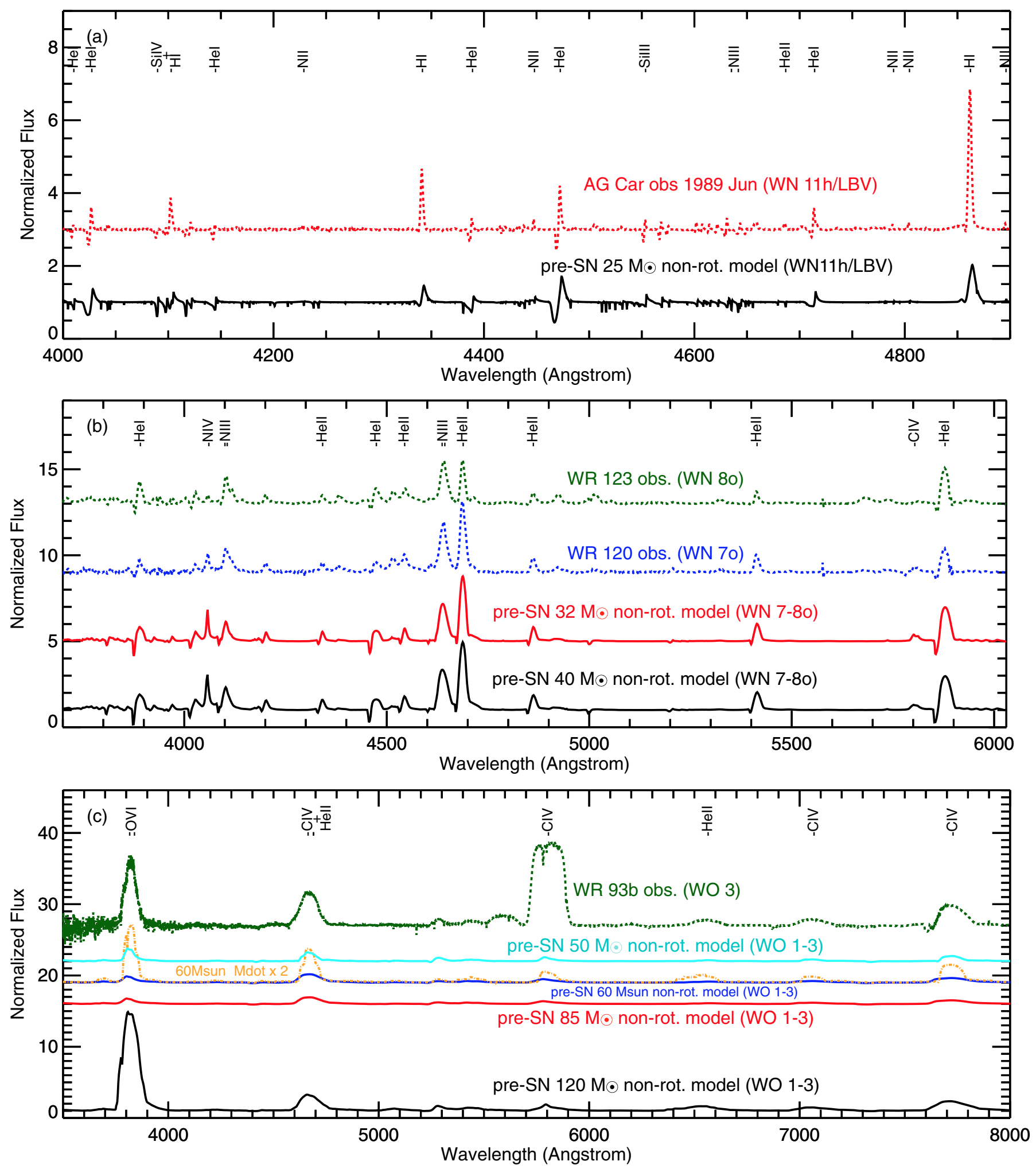

Fig. 2. Montage of the synthetic optical spectra of massive stars at the pre-SN stage from non-rotating stellar evolution models. Observations of stars with similar spectral type (dashed) are shown to support the spectroscopic classifications. The strongest spectral features are indicated. The spectra have been offset in flux for better visualization. a) The $25 M_{\odot}$ model (black), which has a WN11h/LBV spectral type. The 1989 June observations of the LBV AG Car (red) are also shown, when it showed a WN11h spectral type Smith et al. (1994); Stahl et al. (2001); Groh et al. (2009b). b) The $32 M_{\odot}$ (red) and $40 M_{\odot}$ (black) models, which have spectral type WN7-8o, are compared to observations of Galactic WN7o (WR120) and WN8o (WR123) stars, form the catalog of Hamann et al. (1995). c) The $50 M_{\odot}$ (cyan), $60 M_{\odot}$ (blue), $85 M_{\odot}$ (red), and $120 M_{\odot}$ (black) models, which have WO1-3 spectral type. The spectrum of the $60 M_{\odot}$ model with $\dot{M}$ enhanced by a factor of two at the pre-SN stage is shown (orange dot-dashed). The optical spectrum of the Galactic WO 3 star WR 93b (green, from Drew et al. 2004) is also displayed. 

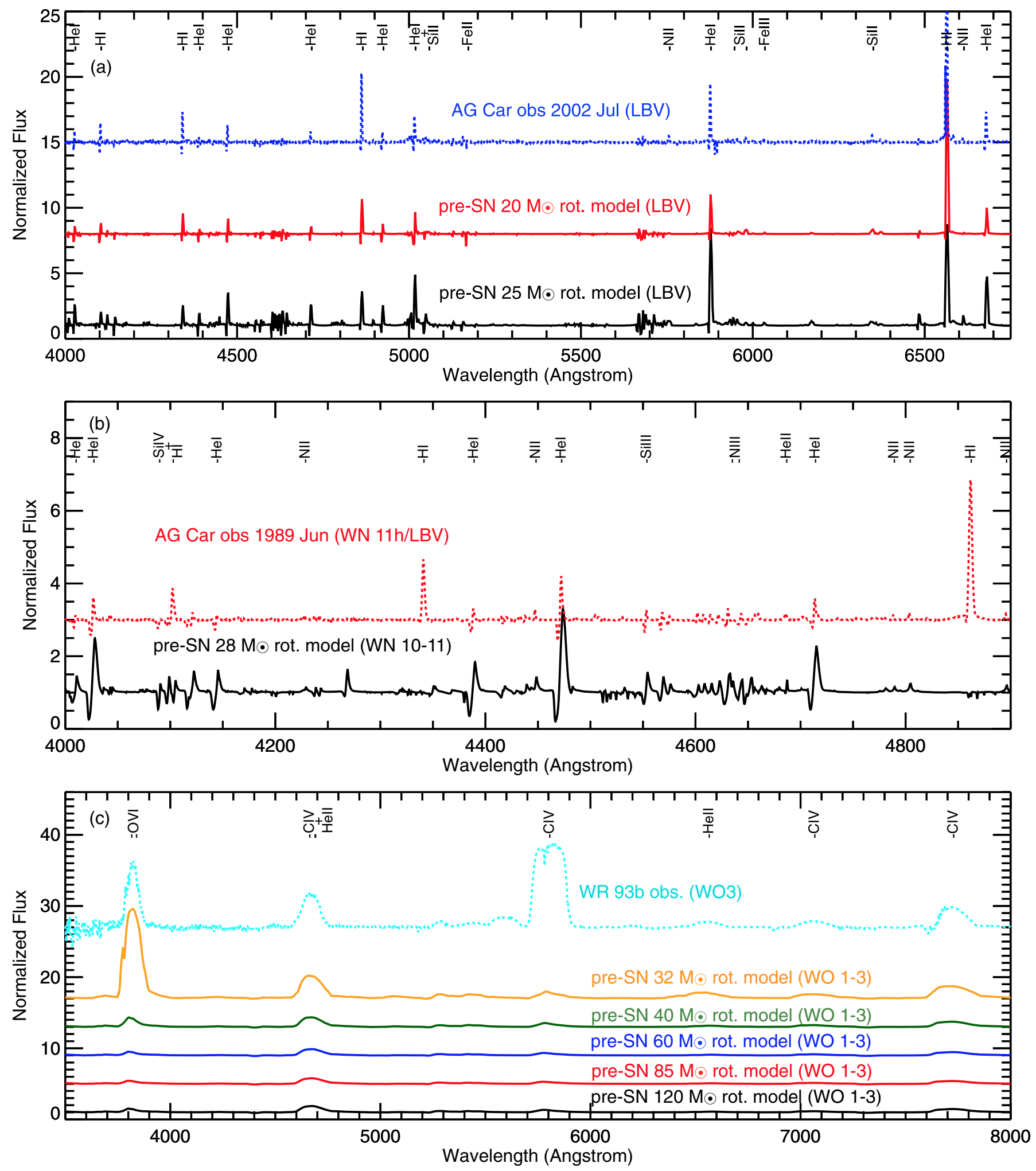

Fig. 3. Similar to Fig. 2, but for rotating models at the pre-SN stage. a) The $20 M_{\odot}$ (red) and $25 M_{\odot}$ models (black), which have spectra similar to LBVs (see also Groh et al. 2013). The 2002 July observations of the LBV AG Car (green) from Groh et al. (2009b) are also show. Note that at that time, AG Car did not show a WN11h spectral type, since it had $T_{\text {eff }} \simeq 16400 \mathrm{~K}$ (Groh et al. $2009 \mathrm{~b}$ ). b) The $28 M_{\odot}$ model (black) compared to the observations of AG Car in 1989 June (from Stahl et al. 2001, when it had a WN11h spectral type). c) The $32 M_{\odot}$ (orange), $40 M_{\odot}$ (green), $60 M_{\odot}$ (blue), $85 M_{\odot}$ (red), and $120 M_{\odot}$ (black) models, which have WO1-3 spectral type. The optical spectrum of the Galactic WO 3 star WR 93b (cyan) is also displayed. 
of observed Galactic WO stars (Drew et al. 2004; Sander et al. 2012). Since the model has not been fiddled to fit the observations, several differences are readily noticeable between the observed and synthetic spectrum. This concerns mostly the C IV $\lambda 5810$ line, which is weaker in the models than in the observations. This is likely caused by the hotter $T_{\text {eff }}$ of the models, which shift the $\mathrm{C}$ ionization structure towards more ionized ions, making C IV $\lambda 5810$ weaker.

The synthetic spectra of the other WO models have much weaker emission lines compared to the observations. This striking result could be interpreted as theoretical evidence for a yet undiscovered population of weak-lined WO stars in the Galaxy. Alternatively, it could be linked to the $\dot{M}$ prescription adopted by the Geneva code and used as input in CMFGEN to compute the synthetic spectra. Nevertheless, we find that models with $\dot{M}$ increased by a factor of 2 (or $v_{\infty}$ decreased by a similar amount) would still produce a WO spectrum, but with line strengths similar to the observations. To illustrate this effect, we show in Fig. 2c the spectrum of the $60 M_{\odot}$ model with $\dot{M}$ increased by a factor of two (orange), while the other parameters are kept fixed. Without further evidence for a larger (or lower) $\dot{M}$ at the WO stage and for consistency with the stellar evolution models, we chose to keep the values of $\dot{M}$ used in the Geneva code and quoted in Table 2.

The optical spectra of the $20 M_{\odot}$ and $25 M_{\odot}$ rotating models are presented in Fig. 3a. They have been discussed in Groh et al. (2013), where we have shown that these stars, at the pre-SN stage, look much alike LBVs such as AG Car (Groh et al. 2009b, 2011), HR Car (Groh et al. 2009a), P Cygni (Najarro et al. 1997; Najarro 2001), and HDE 316285 (Hillier et al. 1998), among others. Their optical spectrum is dominated by strong lines of $\mathrm{H}, \mathrm{He}$ I, N II, and Fe II, often with P-Cygni profiles.

Figure $3 \mathrm{~b}$ shows the optical spectrum of the pre-SN rotating $28 M_{\odot}$ model, which has strong He I lines with P-Cygni profiles. Moderate He II $\lambda 4686$ and Si IV emissions are also present, while N II and N III lines have similar strength. This seems to be a transition spectral morphology between a WN10 and WN11, without the suffix " $h$ " since $\mathrm{H}$ lines are clearly not present.

In Fig. 3c we display the optical spectra of rotating models with $32 M_{\odot}<M_{\text {ini }}<120 M_{\odot}$ at the pre-SN stage. They show broad emission lines of O VI $\lambda 3811$ and C IV $\lambda 5808$, which also implies a WO1-3 classification. The spectral morphology is similar to the non-rotating WO models. The rotating $32 M_{\odot}$ model has a strong-lined spectrum, which resembles the observed Galactic WO stars. The 40, 60, 85 and $120 M_{\odot}$ models have much weaker emission lines. Again, this may be a real effect, which would make challenging to detect the more massive rotating models owing to their weak lines. Alternatively, $\dot{M}$ could be underestimated in the models.

While we did not compute spectra for the models that end up as RSGs, both non-rotating and rotating models become hotter for larger $M_{\text {ini }}$. The relationship between spectral types and $T_{\text {eff }}$ of RSGs has been the subject of debate recently. Using the most recent calibration for Galactic RSGs from Levesque et al. (2005), we find that the SN progenitors from non-rotating stars have spectral types that range from M5 I $\left(9 M_{\odot}\right.$ model $)$ to M1 I (20 $\left.M_{\odot}\right)$, while the spectral type of the rotating models lie between M4.5 I ( $9 M_{\odot}$ model) to M2.5 I (15 $\left.M_{\odot}\right)$. However, the recent results from Davies et al. (2013) casts serious doubts on the validity of the $T_{\mathrm{eff}}-$ spectral type calibration of RSGs, both for the LMC/SMC and for the Galaxy (B. Davies et al., priv. comm.). Therefore, we quote the spectral types of RSGs as "K-M I".
As described in Sect. 4, the rotating $18 M_{\odot}$ model is a yellow luminous star at the pre-SN stage, with $T_{\mathrm{eff}}=5382 \mathrm{~K}$ and $L_{\star}=1.5 \times 10^{5} L_{\odot}$. We shall comment on its spectral type, even though we are not able to compute a synthetic spectrum for this model since its $T_{\text {eff }}$ is too low for a CMFGEN analysis. Stars finishing their lives as yellow luminous stars have been customarily classified as yellow supergiants (YSG); see e.g. Elias-Rosa et al. (2009, 2010); Fraser et al. (2010); Maund et al. (2011); Georgy (2012). However, we suggest here that some of these stars are actually yellow hypergiants (YHG). YSGs and YHGs share a similar region of the HR diagram, having similar $T_{\text {eff }}$ and $L_{\star}$ (de Jager 1998). The main difference is that, contrary to YSGs, YHGs have $\mathrm{H} \alpha$ emission and broader absorption lines. This points out to the presence of a strong mass outflow and an unstable, extended atmosphere close to the Eddington limit in YHGs. Quoted values of $\dot{M}$ of YHGs range from 0.2 to $2 \times 10^{-5} M_{\odot} \mathrm{yr}^{-1}$ during quiescence (de Jager 1998), as in the case of $\rho$ Cassiopeiae (Lobel et al. 2003), IRC +10420 (Driebe et al. 2009), and W4 in Westerlund 1 (Dougherty et al. 2010). Here we find that the $18 M_{\odot}$ rotating model has a quite strong wind at the pre-SN phase with $\dot{M}=1.7 \times 10^{-5} M_{\odot} \mathrm{yr}^{-1}$. This is well in line with the values quoted above for YHGs, which makes it very likely that an extended atmosphere is present. In addition, the model is also extremely bright (Sect. 7). Therefore, we propose a YHG classification with spectral type $\mathrm{G}^{\mathrm{Ia}}{ }^{+}$following the $T_{\text {eff }}$ vs. spectral type calibration of Kovtyukh (2007).

\subsection{SN types associated with the progenitors}

Now that we have determined the spectral type of core-collapse SN progenitors in the initial mass range of 9-120 $M_{\odot}$ we would like to know to which kind of SN they lead to. Ideally, one would need to use the progenitor structure from our stellar structure models as input for hydrodynamical and radiative transfer simulations of the SN (if one indeed occurs). This is well beyond the scope of this paper, and here we use the chemical structure of the progenitor (in particular $\mathrm{H}$ and $\mathrm{He}$ ) as a diagnostic for the SN type. As has been discussed by others (e.g., Heger et al. 2003; Georgy et al. 2009; Dessart et al. 2011; Eldridge et al. 2013), this is a challenging task, since it is not fully understood how much $\mathrm{H}$ exists in the ejecta of SN IIP, IIL, and IIb, and the He content in SN Ib and Ic. Here, we assume the initial mass ranges for the different kind of SNe determined by Georgy et al. (2012), using the chemical abundance criteria as noted in Sect. 3 of the present paper. The analysis that follows below has also the caveat that we assume that all core collapse events lead to a SN, even if a black hole is formed. In addition, as shown by Georgy (2012), mass loss during the RSG phase has a significant impact in the position of the star in the HR diagram at the pre-SN stage. Here, we analyze models only with the standard mass-loss rate during the RSG phase as described in Ekström et al. (2012).

Our results are summarized in Fig. 4, which presents the different channels that link the spectral type of SN progenitors to the core-collapse SN types. The mass ranges of progenitors leading to the different SN types are also shown in parenthesis. In some cases, it is possible that progenitors with different spectral types lead to the same SN type. We then assume a Salpeter-type initial mass function to obtain the fraction of the $\mathrm{SN}$ progenitors that come from the different channels.

We first discuss the results of the rotating models. We obtained that $100 \%$ of the SN IIP progenitors are RSGs. For the $\mathrm{SN}$ IIL/b progenitors, we found that $44 \%$ of them are YHGs while $56 \%$ are LBVs. Our definition supposes that SN progenitors with $4500<T_{\mathrm{eff}}<7000 \mathrm{~K}$ are YHGs and, since there is an 


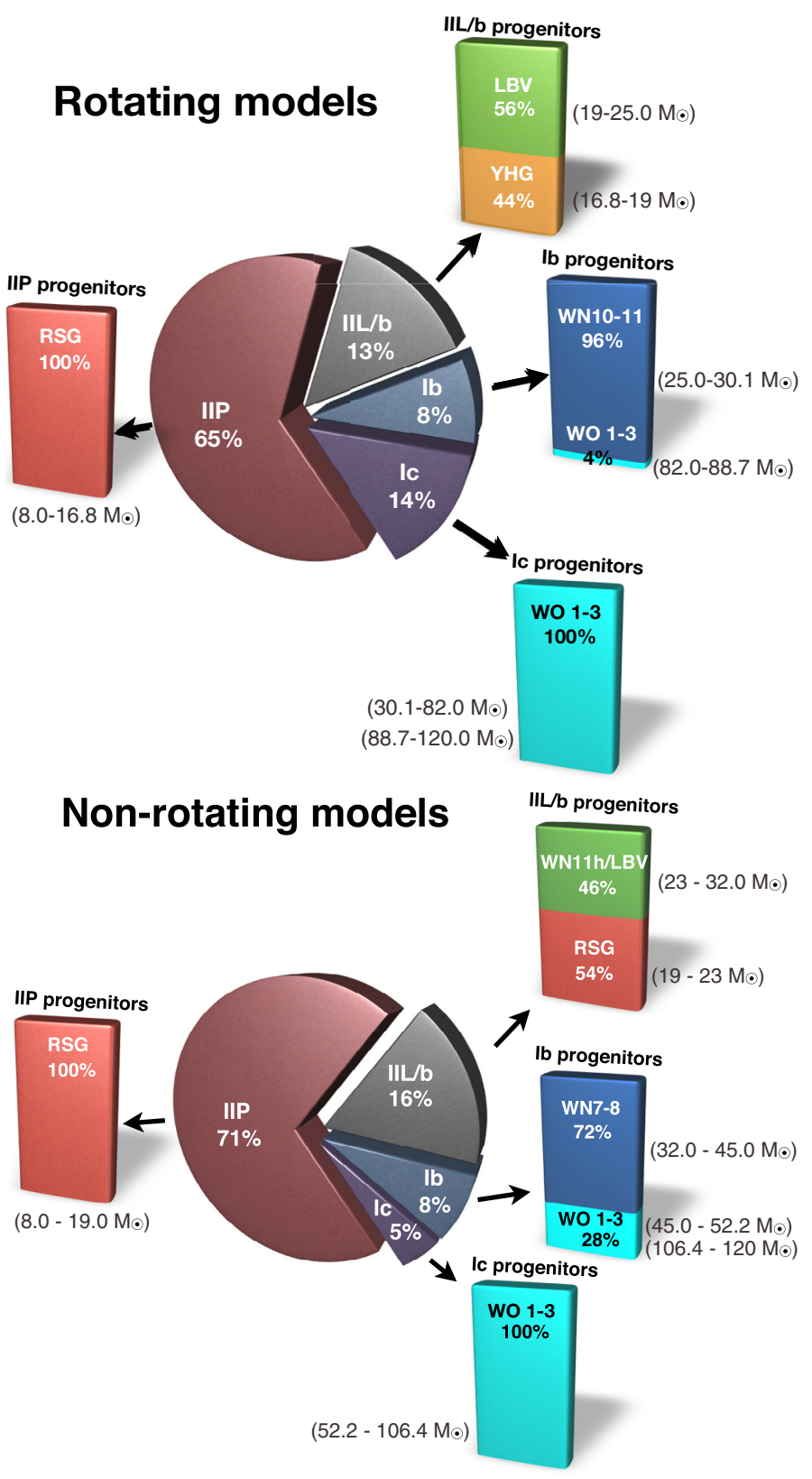

Fig. 4. Diagram illustrating, for different core-collapse SN types, their relative rates and the types of progenitors and their respective frequencies. Initial mass ranges (indicated in parenthesis) and SN types are based on the criteria outlined in Georgy et al. (2012), assuming that the minimum amount of $\mathrm{He}$ in the ejecta to produce a $\mathrm{SN} \mathrm{Ib}$ is $0.6 M_{\odot}$. The upper and lower panels refer to rotating and non-rotating models, respectively.

overlap in spectral type between LBVs and YHGs, we stress that the numbers above are indicative only. The progenitors of SN Ib are in the vast majority WN 10-11 stars, with only $4 \%$ coming from WO stars. Our models indicate that the SN Ic progenitors are all WO stars.

Interestingly, the $25 M_{\odot}$ model, which has an LBV spectrum, is at the transition where stars lose all the $\mathrm{H}$ envelope. It represents likely a transition between an SN IIL/b and SN Ib. It thus seems that there is a parameter space where a small fraction of stars with spectral type similar to LBVs could explode as SN Ib. This is of interest to explaining the progenitor of SN 2006jc, which had an outburst a few years before core collapse was a type Ibn (Pastorello et al. 2007).

For the non-rotating models, the nature of the SN IIP progenitors is unchanged, with all of them being RSGs. However, we found that SN IIL/b progenitors could come from RSGs (54\%) or WN11h/LBV stars (46\%). The nature of the SN Ib progenitors is changed, but the majority of the progenitors are still late WN (WN 7-8) stars, and 28\% are WO stars. The nature of the SN Ic progenitors is unchanged as compared to rotating models, with $100 \%$ of the progenitors being WO stars. These stars present a WO-type spectrum during only a very short period preceding their core collapse, therefore this finding is not in contradiction with the fact that WO stars are very rare stars (see Sect. 9).

\subsection{Linking the interior properties of SN progenitors and their spectra}

Having determined the SN type associated with each progenitor spectral type, let us investigate how the interior structure of SN progenitors is related to their spectra. Figure 5 presents in a schematic way both the spectral appearances of five selected supernova progenitors (upper panel), together with information about their internal structures (lower panel).

Looking at the structure, we readily note that despite their different surface properties and spectra, all progenitor models have an $\mathrm{He}$-free core extending over a radius of a few hundredths of $R_{\odot}$. The outer layers of the He-free cores are made up of $\mathrm{C}$ and $\mathrm{O}$ produced by the He-burning reactions. For this reason, this region is called the carbon-oxygen core. Of course the very central part of this zone is no long composed of $\mathrm{C}$ and $\mathrm{O}$, since the advanced stages have further processed these elements into heavier species.

We see that the carbon-oxygen cores vary in size from two to nine times the radius of the Earth when going from the progenitor that is a RSG to the one that is a WO. At this stage, this part of the star is already sustained at least in part by electron degenerate pressure (remind that a white dwarf has typically the mass of the Sun locked into a sphere having the radius of the Earth). These cores have masses well above the Chandrasekhar mass and are on the verge of collapsing under the effect of their own gravity.

Let us now consider the three models associated to the LBV, YHG and RSG progenitors. Interestingly, the radii of the $\mathrm{H}$-free cores (i.e. He cores) are very similar in the three models and are equal to a few tenths of solar radius. As a rule of thumb, we see that the $\mathrm{H}$-free core is roughly one order of magnitude larger than the He-free core.

In contrast with the cores, the envelopes of the these three progenitors differ greatly. While in the RSG progenitor, the envelope contains more than $6 M_{\odot}$ and is quite H-rich, the one in the YHG comprises only a fraction of a solar mass and is much less $\mathrm{H}$ rich. The trend continues with a decrease of the mass of the envelope and its $\mathrm{H}$ content when one passes from the YHG to the LBV progenitor.

Strikingly, the extensions of the envelopes of the RSG and YHG are not so different and are equal to a few hundreds of solar radii, while as we have just noticed their masses and $\mathrm{H}$ contents are very different. This also illustrates the fact that removing a large part of the $\mathrm{H}$-envelope and reducing significantly its $\mathrm{H}$ content is not sufficient to make the star evolve back to the blue part of the HR diagram. For this to occur, strong mass loss is needed, so the $\mathrm{H}$ content of the envelope is reduced to a few hundredths of $M_{\odot}$. This is well confirmed by many previous works 
Jose H. Groh et al.: Fundamental properties of core-collapse SN progenitors
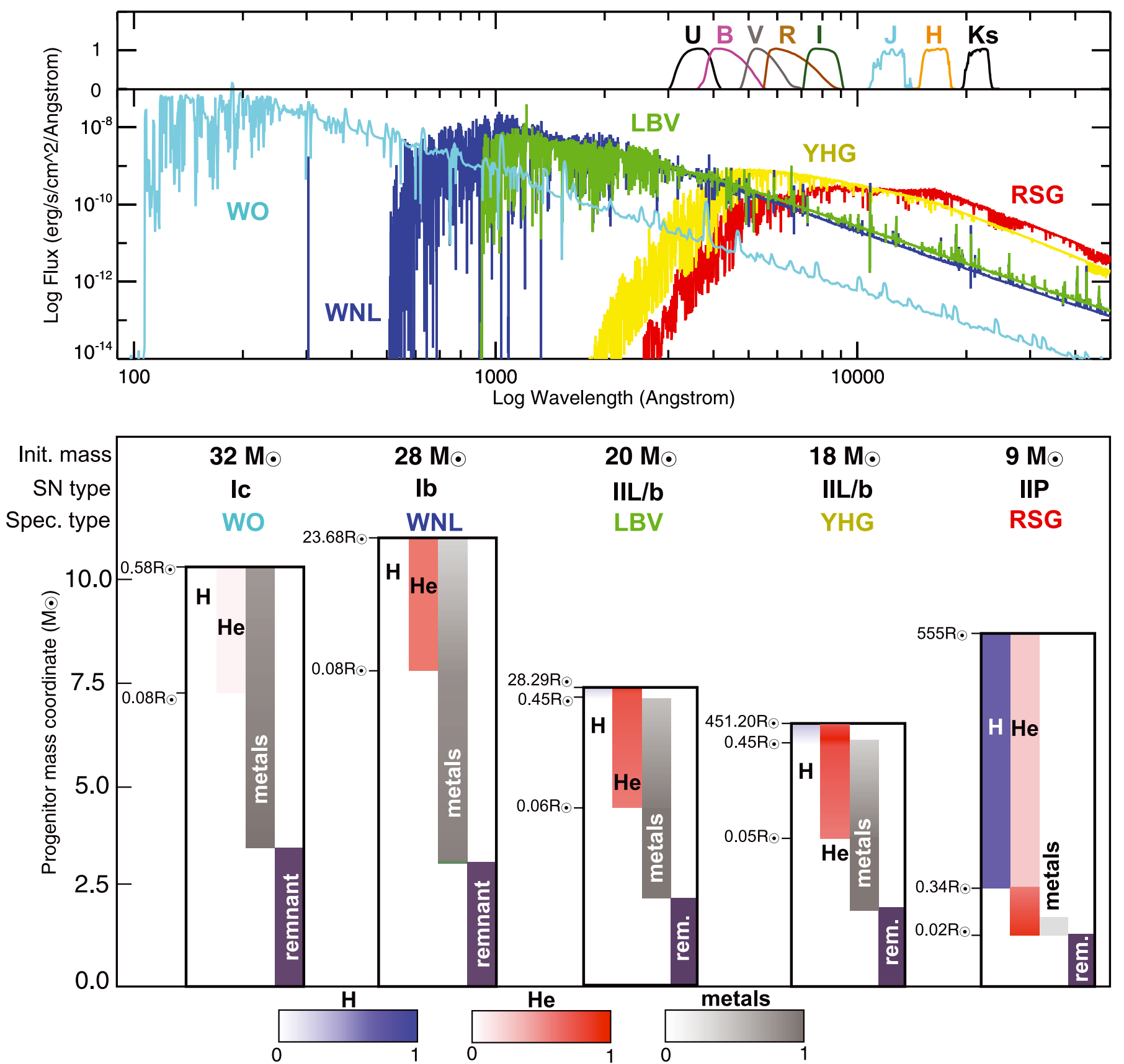

Fig. 5. Top: spectral energy distribution of selected models, showing a WO star with $T_{\text {eff }} \simeq 154000 \mathrm{~K}$ (our $32 M_{\odot}$ rotating mode; cyan), a WNL star of spectral type WN10-11 with $T_{\text {eff }} \simeq 26800 \mathrm{~K}\left(28 M_{\odot}\right.$ rotating model; blue), and an LBV with $T_{\text {eff }} \simeq 20000 \mathrm{~K}$ ( $20 M_{\odot}$ rotating model; green ). We stress that we do not compute model spectra of RSG and YHGs in this paper. To illustrate the SED of these objects, we also overplot MARCS model spectra of a RSG with $T_{\text {eff }}=3600 \mathrm{~K}$ and luminosity scaled to $L_{\star}=1.2 \times 10^{5} L_{\odot}$ (red), and of a YHG with $T_{\text {eff }}=5250 \mathrm{~K}$ and luminosity scaled to $L_{\star}=1.5 \times 10^{5} L_{\odot}$ (yellow). All fluxes have been arbitrarily scaled to a distance of $1 \mathrm{kpc}$. The upper inset shows the normalized bandpasses of the $U B V R I J H K_{\mathrm{S}}$ filters. Bottom: schematic illustration of the interior structure of the SN progenitors for which spectra are shown in the upper panel. We show the Lagrangian mass coordinate of the progenitor in the y axis and the extension of the layers for different chemical elements $(\mathrm{H}$, $\mathrm{He}$, metals) and the baryonic remnant mass (computed as in Hirschi et al. 2005). The radius of selected shells is indicated on the left side of each subpanel, while the spectral type, SN type, and initial mass of the progenitors are indicated immediately above the interior structure. The chemical abundances (by mass) are color-coded in blue $(\mathrm{H})$, red $(\mathrm{He})$, and gray (metals).

(Giannone 1967; Vanbeveren et al. 1998; Salasnich et al. 1999; Yoon \& Cantiello 2010; Georgy 2012; Meynet et al. 2013).

As said above, if the reduction of the H-rich envelope continues, the star will evolve in the blue becoming an LBV or a WR star. We see that our LBV progenitor has a structure not so different from the YHG except for the fact that the LBV has a reduced $\mathrm{H}$-rich envelope.
The LBV and WNL progenitors have similar structures and similar radii, while a drastic change in radius occurs when one passes from the WNL to the WO progenitor. Note that $\mathrm{H}$ content of the outer layers seems to be one of the key factors leading to these various supernovae progenitors. The sequence of models from right to left corresponds to a decrease of $\mathrm{H}$ in the envelope. It seems that the passage from red to blue (and from blue to very 
Table 3. Surface chemical composition and comparison of spectral types of core-collapse SN progenitor with previous studies.

\begin{tabular}{|c|c|c|c|c|c|c|c|c|}
\hline $\begin{array}{l}M_{\text {ini }} \\
\left(M_{\odot}\right)\end{array}$ & $\begin{array}{c}X_{\text {surf }} \\
\text { (mass) }\end{array}$ & $\begin{array}{c}Y_{\text {surf }} \\
\text { (mass) }\end{array}$ & $\begin{array}{c}C_{\text {surf }} \\
\text { (mass) }\end{array}$ & $\begin{array}{c}N_{\text {surf }} \\
\text { (mass) }\end{array}$ & $\begin{array}{c}O_{\text {surf }} \\
\text { (mass) }\end{array}$ & $\begin{array}{l}\text { Previous Sp. Type }{ }^{a} \\
\text { (Chem. Abund.) }\end{array}$ & New Sp. Type & SN type ${ }^{a}$ \\
\hline \multicolumn{9}{|c|}{ non-rotating models } \\
\hline 9 & 0.68 & 0.31 & $1.6 \times 10^{-3}$ & $3.0 \times 10^{-3}$ & $4.4 \times 10^{-3}$ & RSG & $K-M I$ & IIP \\
\hline 12 & 0.68 & 0.31 & $1.3 \times 10^{-3}$ & $2.5 \times 10^{-3}$ & $4.8 \times 10^{-3}$ & RSG & $K-M I$ & IIP \\
\hline 15 & 0.65 & 0.34 & $1.1 \times 10^{-3}$ & $3.2 \times 10^{-3}$ & $4.4 \times 10^{-3}$ & RSG & $K-M I$ & IIP \\
\hline 20 & 0.48 & 0.51 & $8.4 \times 10^{-5}$ & $6.9 \times 10^{-3}$ & $1.6 \times 10^{-3}$ & RSG & $K-M I$ & $\mathrm{IIL} / \mathrm{b}$ \\
\hline 23 & 0.31 & 0.67 & $6.4 \times 10^{-5}$ & $8.1 \times 10^{-3}$ & $1.5 \times 10^{-4}$ & RSG & $K-M I$ & $\mathrm{IIL} / \mathrm{b}$ \\
\hline 25 & 0.16 & 0.83 & $6.8 \times 10^{-5}$ & $8.2 \times 10^{-3}$ & $1.1 \times 10^{-4}$ & WNL & WN11h/LBV & $\mathrm{IIL} / \mathrm{b}$ \\
\hline 32 & 0.00 & 0.99 & $1.4 \times 10^{-4}$ & $8.1 \times 10^{-3}$ & $1.0 \times 10^{-4}$ & WNE & WN 7-8o & $\mathrm{Ib}$ \\
\hline 40 & 0.00 & 0.99 & $9.5 \times 10^{-5}$ & $8.2 \times 10^{-3}$ & $9.2 \times 10^{-5}$ & WNE & WN 7-8o & $\mathrm{Ib}$ \\
\hline 50 & 0.00 & 0.19 & 0.46 & $1.5 \times 10^{-5}$ & 0.33 & WC & WO $1-3$ & $\mathrm{Ib}$ \\
\hline 60 & 0.00 & 0.27 & 0.51 & 0.00 & 0.20 & WC & WO $1-3$ & Ic \\
\hline 85 & 0.00 & 0.25 & 0.49 & 0.00 & 0.25 & WC & WO $1-3$ & Ic \\
\hline 120 & 0.00 & 0.24 & 0.46 & 0.00 & 0.29 & WC & WO 1-3 & $\mathrm{Ib}$ \\
\hline \multicolumn{9}{|c|}{ rotating models, initial $v_{\text {rot }} / v_{\text {crit }}=0.4$} \\
\hline 9 & 0.64 & 0.35 & $6.2 \times 10^{-4}$ & $3.8 \times 10^{-3}$ & $4.4 \times 10^{-3}$ & RSG & $K-M I$ & IIP \\
\hline 12 & 0.64 & 0.35 & $6.2 \times 10^{-4}$ & $3.9 \times 10^{-3}$ & $4.2 \times 10^{-3}$ & RSG & $K-M I$ & IIP \\
\hline 15 & 0.56 & 0.43 & $4.9 \times 10^{-4}$ & $4.7 \times 10^{-3}$ & $3.5 \times 10^{-3}$ & RSG & $K-M I$ & IIP \\
\hline 16.5 & 0.55 & 0.44 & $4.8 \times 10^{-4}$ & $4.8 \times 10^{-3}$ & $3.4 \times 10^{-3}$ & RSG & $K-M I$ & IIP \\
\hline 18 & 0.38 & 0.61 & $4.9 \times 10^{-5}$ & $7.0 \times 10^{-3}$ & $1.4 \times 10^{-3}$ & RSG & $G 1 \mathrm{Ia}^{+}$ & IIL/b \\
\hline 20 & 0.24 & 0.75 & $6.2 \times 10^{-5}$ & $7.8 \times 10^{-3}$ & $4.9 \times 10^{-4}$ & WNL & LBV & $\mathrm{IIL} / \mathrm{b}$ \\
\hline 25 & 0.03 & 0.93 & $1.6 \times 10^{-4}$ & $1.6 \times 10^{-3}$ & $6.5 \times 10^{-4}$ & WNL & LBV & $\mathrm{Ib}$ \\
\hline 28 & 0.00 & 0.97 & $9.3 \times 10^{-3}$ & $1.0 \times 10^{-2}$ & $1.2 \times 10^{-3}$ & WNL & WN $10-11$ & $\mathrm{Ib}$ \\
\hline 32 & 0.00 & 0.28 & 0.47 & $2.2 \times 10^{-3}$ & 0.23 & WC & WO 1 & Ic \\
\hline 40 & 0.00 & 0.28 & 0.50 & $4.0 \times 10^{-5}$ & 0.20 & WC & WO 1-2 & Ic \\
\hline 60 & 0.00 & 0.28 & 0.49 & 0.00 & 0.21 & WC & WO $1-3$ & Ic \\
\hline 85 & 0.00 & 0.26 & 0.47 & 0.00 & 0.25 & WC & WO 1-3 & $\mathrm{Ib}$ \\
\hline 120 & 0.00 & 0.26 & 0.49 & 0.00 & 0.23 & WC & WO 1 & Ic \\
\hline
\end{tabular}

Notes. ${ }^{(a)}$ From Georgy et al. (2012).

blue) does not occur gradually but rather in a very sharp manner when some limit in the $\mathrm{H}$ surface content is reached.

\section{Spectroscopic vs. chemical abundance classifications of massive stars}

We computed here, for the first time, the fundamental parameters and observables of SN progenitors across a broad mass range, based on evolutionary calculations. Not surprisingly, having a spectrum allows a much more precise determination of the spectral type of the SN progenitors, using the same observational criteria employed for classifying the spectra of real stars. This allows a refined understanding of which kind of single stars will be progenitors of the different types of core-collapse SNe.

In previous studies, the properties of core-collapse SN progenitors, such as their spectral type, were estimated according to the surface chemical abundances, luminosity, and temperature (Georgy et al. 2009, 2012). These were accomplished using the relationship between spectral types and surface abundances from Smith \& Maeder (1991) and Meynet \& Maeder (2003), as follows:

- RSGs have $\log \left(T_{\text {eff }} / \mathrm{K}\right)<3.66$;

- O stars have a surface hydrogen mass fraction $X_{\mathrm{H}}>0.3$ and $\log \left(T_{\text {eff }} / \mathrm{K}\right)>4.5$;

- WR stars have $\log \left(T_{\text {eff }} / \mathrm{K}\right)>4.0$ and $X_{\mathrm{H}}<0.3$;

- WNL stars are WR stars with $X_{\mathrm{H}}>10^{-5}$.

- WNE stars have $X_{\mathrm{H}}<10^{-5}$ and a $C$ surface abundance $X_{\mathrm{C}}$ lower than the 0.1 times the $\mathrm{N}$ surface abundance $X_{\mathrm{N}}$;

- WNC stars have $X_{\mathrm{H}}<10^{-5}$ and $\frac{X_{\mathrm{C}}}{X_{\mathrm{N}}}=0.1$ to 10 ;
- WC stars have $X_{\mathrm{H}}<10^{-5}, X_{\mathrm{C}}>X_{\mathrm{N}}$, and surface abundances (by number) such as $\frac{\mathrm{C}+\mathrm{O}}{\mathrm{He}}<1$;

- WO stars have $X_{\mathrm{H}}<10^{-5}, X_{\mathrm{C}}>X_{\mathrm{N}}$, and $\frac{\mathrm{C}+\mathrm{O}}{\mathrm{He}}>1$.

In Table 3 we show how our refined spectral type classifications compared to those from Georgy et al. (2012). The abundances at the surface, which were the main criterium used in previous evolutionary studies, are also shown. Important differences between our spectral type determination and previous ones can be readily noted, and we comment on them below.

Starting from models with the lowest initial masses, we note that RSGs were not classified in spectroscopic subtypes in previous stellar evolution studies. For the 20 and $25 M_{\odot}$ rotating models, we have determined that a spectral type reminiscent of LBVs is found. However, we see that a spectral classification of a late-type WN star (WNL) was suggested before. This was because, in the absence of a spectrum, it was assumed that stars with $T_{\text {eff }}>20000 \mathrm{~K}$ and $\mathrm{H}$ surface abundance $\left(X_{\text {surf }}\right)$ greater than $10^{-5}$ would be WNL stars (Meynet \& Maeder 2003; Georgy et al. 2012). In Groh et al. (2013) we showed that the 20 and $25 M_{\odot}$ rotating models actually resemble LBVs rather than WNL stars. The WNLs with the latest possible spectral type (WN11h) have $T_{\text {eff }} \gtrsim 25000 \mathrm{~K}$, which is well above the $T_{\mathrm{eff}} \simeq 20000 \mathrm{~K}$ found for the 20 and $25 M_{\odot}$ rotating models at the pre-SN stage. The non-rotating $25 M_{\odot}$ model is a transition between an LBV and a WN11h spectral type.

For higher initial masses, we show here that a WN 7-8 spectral type is found for the 32 and $40 M_{\odot}$ non-rotating models, while Georgy et al. (2012) suggested an early-type WN (WNE) classification. This difference stems from the assumption made in previous works that stars with $X_{\text {surf }}<10^{-5}$ would 
be WNE stars. With the spectra computed here, we show that this assumption is not necessarily true, and that WNL stars can also have $X_{\text {surf }}<10^{-5}$. This was also found in previous works that analyzed observed WNL stars, such as WR 123 (Hamann et al. 2006). The main parameter that determines whether the star is a WNE or WNL is $T_{\text {eff }}$, which regulates the He and $\mathrm{N}$ ionization structures in the wind and ultimately sets the spectral type of the star (Hillier 1987).

For the rotating $32-120 M_{\odot}$ and non-rotating 60-120 $M_{\odot}$ models we obtain a WO spectral type. This is also in contrast with previous estimates, that have commonly associated a WC classification to the SN progenitors based on the surface abundances of $\mathrm{C}, \mathrm{O}$, and He. Earlier studies assumed that a WO subtype would only appear when $(\mathrm{C}+\mathrm{O}) / \mathrm{He}>1.0$ (by number) at the surface (Smith \& Maeder 1991), i.e., that a significant $\mathrm{O}$ enrichment would be responsible for the appearance of the WO subtype. Here we find that, once a minimal amount of $\mathrm{O}$ is present at the surface, the WO subtype arises mainly because of ionization effects. The extremely high $T_{\text {eff }}$ of stars at their end is what produces the broad, strong O VI $\lambda 3818$ emission that characterizes the WO subtype. Therefore, we show here that stellar evolution models predict, even at solar metallicity, that WO stars are the end stage of stellar evolution for the most massive stars, at least up to $120 M_{\odot}$. Also, we note that WO stars arise both from non-rotating and rotating stars, although the mass range at which they appear is tighter for non-rotating $\left(60-120 M_{\odot}\right)$ than for rotating stars $\left(32-120 M_{\odot}\right)$. However, since rotating stars are more luminous than non-rotating stars of the same mass, the luminosity range in which WO appears is similar for rotating and non-rotating models.

In a further work, we shall compute the output spectra for all the evolutionary stages from the zero age main sequence to core collapse. This will allow us to provide the evolutionary connections of the spectral types as they are predicted by theoretical models for single stars.

\section{Absolute magnitudes, colors, and bolometric corrections of SN progenitors}

Fortunately, a significant amount of archival high spatial resolution imaging data exists. When a SN occurs, these archival data can be used to search for the SN progenitor and obtain its magnitudes, or at least to derive lower limits. Here, we present a theoretical database to aid the comparison between the endstage of the Geneva evolutionary tracks and observations of SN progenitors. We performed synthetic photometry of our SN progenitor models using the Chorizos code (Maíz-Apellániz 2004), adopting its built-in passband and zero point definitions that were obtained from Cohen et al. (2003); Maíz Apellániz (2006); Holberg \& Bergeron (2006); Maíz-Apellániz (2007).

For a given filter $P$, the absolute magnitudes $\left(M_{P}\right)$ in the modified Vega magnitude system are

$M_{P}=-2.5 \log _{10}\left(\frac{\int P(\lambda) F_{\lambda}(\lambda) \lambda \mathrm{d} \lambda}{\int P(\lambda) F_{\lambda, \mathrm{Vega}}(\lambda) \lambda \mathrm{d} \lambda}\right)+\mathrm{ZP}_{P}$,

where $\lambda$ is the wavelength, $P(\lambda)$ is the sensitivity curve of the system, $F_{\lambda}$ is the model flux at $10 \mathrm{pc}, F_{\lambda \text {,vega }}$ is the flux of Vega scaled to a distance of $10 \mathrm{pc}$, and $\mathrm{ZP}_{P}$ is the zero point.

The bolometric magnitudes $\left(M_{\mathrm{bol}}\right)$ are computed with the usual relationship, assuming that the solar $M_{\mathrm{bol}}$ is $4.74 \mathrm{mag}$ :

$M_{\mathrm{bol}}=-2.5 \log _{10} L_{\star}+4.74$, and bolometric corrections in a given filter $P\left(\mathrm{BC}_{P}\right)$ are then obtained using

$\mathrm{BC}_{P}=M_{\mathrm{bol}}-M_{P}$.

To illustrate the wavelength dependence of the absolute magnitudes and bolometric corrections of our SN progenitor models, we quote them in the following filters: Johnson-Cousins UBVRI, Hubble Space Telescope (HST)/Wide Field Planetary Camera 2 (WFPC2) F170W, F300W, F450W, F606W, F814W, and 2MASS $J, H$, and $K_{\mathrm{S}}$, as can be seen in Tables 4 and 5 .

Figure 5 (top panel) presents the spectral energy distribution of selected SN progenitor models, covering the range in $T_{\text {eff }}$ explored in this work. The behavior of the absolute magnitudes and bolometric corrections as a function of initial mass is then regulated by how much flux from the star falls within the passband of a given filter. The shape of the spectral energy distribution depends on the effective temperature, luminosity, and wind density. Therefore, not necessarily the most massive and luminous stars are the brighter ones in a given filter. This has also been discussed by Eldridge et al. (2007) in the context of the comparison between RSGs and super Asymptotic Giant Branch stars. Figure 6 shows the absolute magnitudes in the WFPC2/F170W and $U B V R I J H K_{\mathrm{S}}$ filters as a function of initial mass. The symbols are coded according to the progenitor spectral type, ensuing SN type, and the evolutionary model type used (rotating or non-rotating).

Let us look at the absolute magnitudes and BCs of the SN progenitors and how they broadly vary according to the initial mass and wavelength. In general, we find that the RSGs (red symbols) are bright in the RIJHK filters and faint in the $F 170 \mathrm{~W}$ and $U B$ filters. LBVs (green symbols), YHGs (yellow), and WNs (blue) are relatively bright in all filters, while WOs (cyan) are faint in all filters, with the exception of the $F 170 \mathrm{~W}$ filter.

We obtain that the brightest SN progenitors in the $F 170 \mathrm{~W}$ filter are the $20 M_{\odot}(\mathrm{LBV}), 25 M_{\odot}(\mathrm{LBV})$, and $28 M_{\odot}(\mathrm{WN} 10$ 11) rotating models, and the 32 and $40 M_{\odot}$ non-rotating models (WN7-8o). They are followed in brightness by the 32-120 $M_{\odot}$ rotating models and 50-120 non-rotating models, which are WOs. The RSGs are the faintest progenitors in these filters.

In the $U$ and $B$ bands, the brightest models are the 18, 20, 25 , and $28 M_{\odot}$ rotating models, which have $M_{U}=-6.05,-7.92$, -8.30 , and $-7.83 \mathrm{mag}$, respectively (Fig. 6). They are followed by the 25,32 , and $40 M_{\odot}$ non-rotating models (WN8, $M_{U}=$ $-7.47,-6.76$, and -7.09 , respectively). In the $U$-band, the 32 and $120 M_{\odot}$ rotating models (WOs) are brighter than the rotating 9-15 $M_{\odot}$ models (RSGs), while the situation is reversed in the redder filters. This is explained by the huge difference in $T_{\text {eff }}$ between WOs ( 145 000-175000 K) and RSGs ( 3480-3740 K). While the earlier emits the bulk of their flux in the far-UV, RSGs emit most of the flux in the near-IR (Fig. 5). The WO stars have similar absolute magnitudes, with the exception of the nonrotating $120 M_{\odot}$ model, which is significantly more luminous ( $~ 0.8-1.3 \mathrm{mag}$ ) than the other WO models.

In the $V$-band, we predict that the brightest $\mathrm{SN}$ progenitors should be the $18 M_{\odot}$ rotating models (YHG) and $23 M_{\odot}$ nonrotating model (RSG). They are followed by the $20 M_{\odot}$ and $25 M_{\odot}$ rotating models, and by the non-rotating $20 M_{\odot}$ model. In the $R$ - and $I$-bands, RSGs are the brightest progenitors, followed by YHGs, LBVs, WN8s, and WOs. In the near-infrared $J H K_{\mathrm{S}}$, RSGs are even brighter than the other classes of progenitors.

For progenitors in the RSG regime $\left(M_{\text {ini }}=9-15 M_{\odot}\right)$, the non-rotating models are systematically fainter than the rotating models at the same initial mass. The difference in magnitude can be up to $0.7 \mathrm{mag}$, as in the case of the $15 M_{\odot}$ model in 
Table 4. Absolute magnitudes of the SN progenitor models in different filters: Johnson-Cousins UBVRI, HST/WFPC2 F170, F336W, F450W, $F 555 W, F 606 W$, and $F 814 W$, and $2 \mathrm{MASS} J H K_{\mathrm{S}}$.

\begin{tabular}{|c|c|c|c|c|c|c|c|c|c|c|c|c|c|c|c|}
\hline $\begin{array}{l}M_{\text {ini }} \\
\left(M_{\odot}\right)\end{array}$ & pe & $\begin{array}{l}\Lambda_{U} \\
\mathrm{agg})\end{array}$ & $\begin{array}{c}M_{B} \\
(\mathrm{mag})\end{array}$ & $\begin{array}{c}M_{V} \\
(\mathrm{mag})\end{array}$ & $\begin{array}{c}M_{R} \\
(\mathrm{mag})\end{array}$ & $\begin{array}{c}M_{I} \\
(\mathrm{mag})\end{array}$ & $\begin{array}{l}M_{170} \\
(\mathrm{mag})\end{array}$ & $\begin{array}{c}M_{336} \\
(\mathrm{mag})\end{array}$ & $\begin{array}{c}M_{450} \\
(\mathrm{mag})\end{array}$ & $\begin{array}{c}M_{555} \\
\text { (mag) }\end{array}$ & $\begin{array}{l}M_{606} \\
\text { nag) }\end{array}$ & $\begin{array}{l}M_{814} \\
(\mathrm{mag})\end{array}$ & $\begin{array}{c}M_{J} \\
(\mathrm{mag})\end{array}$ & $\begin{array}{c}M_{H} \\
(\mathrm{mag})\end{array}$ & $\begin{array}{c}M_{K} \\
(\mathrm{mag})\end{array}$ \\
\hline \multicolumn{16}{|c|}{ non-rotating models } \\
\hline 9 & $M I$ & .67 & -2.65 & -4.21 & -5.41 & -6.98 & -1.77 & -0.43 & -3.04 & -4.19 & -4.79 & -6.92 & -8.51 & -9.43 & -9.71 \\
\hline 12 & & .95 & 86 & -4.8 & & -7.25 & -2.08 & & -3.57 & -4.81 & & -7.19 & & & -9.82 \\
\hline 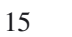 & & 57 & 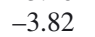 & 5.40 & 656 & -7.86 & -2.70 & & -4.24 & & & -7.81 & & & -10.40 \\
\hline 20 & & .54 & .02 & -6.76 & -7.6 & -8.67 & -3.60 & & -5.46 & & & & & & -10.97 \\
\hline 23 & $M I$ & 1.25 & -6.41 & -7.90 & -8.56 & -9.14 & -4.28 & & & & & & & -10.63 & -10.78 \\
\hline 25 & & & & & & & & & & & & & & & \\
\hline 32 & & & & 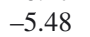 & & & & & & & & & & & \\
\hline 40 & & & & & & & & & & & & & & & \\
\hline$\ldots$ & & & & & & & & & & & & & & & \\
\hline 60 & & 9 & 2.6 & -2 & -2 & -2.71 & & -4 & -2 & -2 & 2 & -2 & & 7 & -3.29 \\
\hline 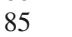 & & & & & & & & & & & & & & & -3.82 \\
\hline & & & & & & -4.20 & -7.65 & -5.89 & -4.70 & & & & & & \\
\hline \multicolumn{16}{|c|}{ rotating models, initial $v_{\text {rot }} / v_{\text {crit }}=0.4$} \\
\hline 9 & & & & & & -7.13 & & & -3.36 & & & & & & 76 \\
\hline 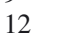 & & 44 & 3.6 & -5 . & & & & & -4 & & & & & & -10.31 \\
\hline 15 & $M I$ & .11 & -4.46 & -6.17 & -7.18 & -8.36 & -3.23 & -1.89 & -4 . & -6.18 & -6 & -8 & -9.70 & & -10.81 \\
\hline 16 & & & & & & & & & & & & & & & \\
\hline 18 & & & & $-\varepsilon$ & & -8 & -4 & & -7 & -8 & $-\varepsilon$ & $-\varepsilon$ & -8 & -9.83 & -9.91 \\
\hline 20 & & .92 & -6.9 & -6 & 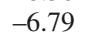 & -6.71 & -8 & -8.37 & -6 & -6.81 & & & & & -6.90 \\
\hline 25 & & & & & & & & & & & & & & 7 & \\
\hline 28 & & & & & & & & & & & & & & & \\
\hline 32 & & & & & & & & & & & & & & & \\
\hline 40 & & & & & & & & & & & & & & 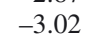 & \\
\hline 60 & & 19 & -3 & -2 . & -2 . & -3 & & -4 & -3 & -2.99 & & & -3 & -3.44 & -3.68 \\
\hline 85 & & & & -3 & & & & & & & & & & & -4.20 \\
\hline 120 & WO1 & .09 & -3.00 & -2.92 & -2.95 & -3.06 & -6.11 & -4.55 & -3.05 & -2.99 & -2.96 & -3.05 & -3.22 & -3.44 & -3.68 \\
\hline
\end{tabular}

Table 5. Bolometric corrections of the SN progenitor models in different filters: Johnson-Cousins UBVRI, HST/WFPC2 F170, F336W, F450W, $F 555 \mathrm{~W}, \mathrm{~F} 606 \mathrm{~W}$, and $F 814 \mathrm{~W}$, and $2 \mathrm{MASS} J H K_{\mathrm{S}}$.

\begin{tabular}{|c|c|c|c|c|c|c|c|c|c|c|c|c|c|c|c|}
\hline $\begin{array}{l}M_{\mathrm{ini}} \\
\left(M_{\odot}\right)\end{array}$ & Sp. Type & $\begin{array}{c}B C_{U} \\
(\mathrm{mag})\end{array}$ & $\begin{array}{c}B C_{B} \\
(\mathrm{mag})\end{array}$ & $\begin{array}{c}B C_{V} \\
(\mathrm{mag})\end{array}$ & $\begin{array}{c}B C_{R} \\
(\mathrm{mag})\end{array}$ & $\begin{array}{c}B C_{I} \\
(\mathrm{mag})\end{array}$ & $\begin{array}{l}B C_{170} \\
(\mathrm{mag})\end{array}$ & $\begin{array}{l}B C_{336} \\
(\mathrm{mag})\end{array}$ & $\begin{array}{l}B C_{450} \\
(\mathrm{mag})\end{array}$ & $\begin{array}{l}B C_{555} \\
(\mathrm{mag})\end{array}$ & $\begin{array}{l}B C_{606} \\
\text { (mag) }\end{array}$ & $\begin{array}{l}B C_{814} \\
(\mathrm{mag})\end{array}$ & $\begin{array}{c}B C_{J} \\
(\mathrm{mag})\end{array}$ & $\begin{array}{c}B C_{H} \\
(\mathrm{mag})\end{array}$ & $\begin{array}{c}B C_{K} \\
(\mathrm{mag})\end{array}$ \\
\hline \multicolumn{16}{|c|}{ non-rotating models } \\
\hline 9 & $K-M I$ & -6.08 & -4.10 & -2.54 & -1.34 & 0.23 & -4.98 & -6.32 & -3.71 & -2.56 & -1.96 & 0.17 & 1.76 & 2.68 & 2.96 \\
\hline 12 & $K-M I$ & -5.97 & -3.77 & -2.11 & -1.02 & 0.32 & -4.84 & -6.19 & -3.35 & -2.12 & -1.54 & 0.27 & 1.74 & 2.63 & 2.90 \\
\hline 15 & $K-M I$ & -5.95 & -3.70 & -2.03 & -0.96 & 0.34 & -4.82 & -6.16 & -3.28 & -2.03 & -1.46 & 0.29 & 1.74 & 2.62 & 2.88 \\
\hline 20 & $K-M I$ & -5.68 & -3.20 & -1.46 & -0.53 & 0.45 & -4.61 & -5.94 & -2.75 & -1.45 & -0.92 & 0.42 & 1.70 & 2.51 & 2.75 \\
\hline 23 & $K-M I$ & -4.24 & -2.08 & -0.59 & 0.07 & 0.66 & -4.20 & -5.02 & -1.64 & -0.59 & -0.19 & 0.64 & 1.59 & 2.14 & 2.30 \\
\hline 25 & WN11h/LBV & -1.23 & -2.33 & -2.53 & -2.57 & -2.72 & 0.05 & -0.75 & -2.32 & -2.48 & -2.50 & -2.70 & -2.90 & -2.96 & -2.85 \\
\hline 32 & WN7-8o & -2.42 & -3.57 & -3.70 & -3.72 & -3.70 & -0.51 & -1.90 & -3.55 & -3.66 & -3.67 & -3.70 & -3.72 & -3.63 & -3.48 \\
\hline 40 & WN7-8o & -2.38 & -3.36 & -3.62 & -3.56 & -3.61 & -0.49 & -1.86 & -3.35 & -3.50 & -3.54 & -3.56 & -3.56 & -3.49 & -3.33 \\
\hline 50 & WO1-3 & -5.64 & -6.78 & -6.87 & -6.85 & -6.75 & -3.76 & -5.25 & -6.74 & -6.81 & -6.83 & -6.75 & -6.59 & -6.36 & -6.11 \\
\hline 60 & WO1-3 & -5.68 & -6.78 & -6.89 & -6.86 & -6.76 & -3.63 & -5.25 & -6.73 & -6.81 & -6.85 & -6.76 & -6.62 & -6.40 & -6.18 \\
\hline 85 & WO1-3 & -5.85 & -6.96 & -7.06 & -7.03 & -6.93 & -3.84 & -5.42 & -6.91 & -6.99 & -7.01 & -6.93 & -6.78 & -6.56 & -6.32 \\
\hline 120 & WO1-3 & -4.69 & -6.26 & -6.74 & -6.75 & -6.69 & -3.23 & -5.00 & -6.19 & -6.63 & -6.71 & -6.70 & -6.60 & -6.51 & -6.41 \\
\hline \multicolumn{16}{|c|}{ rotating models, initial $v_{\text {rot }} / v_{\text {crit }}=0.4$} \\
\hline 9 & $K-M I$ & -6.01 & -3.89 & -2.27 & -1.14 & 0.29 & -4.89 & -6.24 & -3.48 & -2.28 & -1.69 & 0.23 & 1.75 & 2.65 & 2.92 \\
\hline 12 & $K-M I$ & -5.98 & -3.79 & -2.14 & -1.05 & 0.32 & -4.85 & -6.20 & -3.38 & -2.15 & -1.57 & 0.26 & 1.74 & 2.64 & 2.90 \\
\hline 15 & $K-M I$ & -5.87 & -3.52 & -1.80 & -0.79 & 0.39 & -4.74 & -6.08 & -3.08 & -1.80 & -1.24 & 0.34 & 1.72 & 2.59 & 2.84 \\
\hline 16 & $K-M I$ & -5.83 & -3.46 & -1.73 & -0.74 & 0.40 & -4.72 & -6.06 & -3.02 & -1.73 & -1.18 & 0.36 & 1.72 & 2.58 & 2.82 \\
\hline 18 & $G 1 I a^{+}$ & -2.17 & -1.11 & -0.11 & 0.34 & 0.74 & -3.89 & -3.08 & -0.81 & -0.11 & 0.16 & 0.74 & 1.30 & 1.60 & 1.68 \\
\hline 20 & LBV & -0.55 & -1.57 & -1.70 & -1.67 & -1.75 & 0.35 & -0.10 & -1.54 & -1.65 & -1.63 & -1.73 & -1.79 & -1.74 & -1.56 \\
\hline 25 & LBV & -0.72 & -1.75 & -1.87 & -1.77 & -1.88 & 0.30 & -0.27 & -1.72 & -1.81 & -1.77 & -1.84 & -1.78 & -1.70 & -1.38 \\
\hline 28 & WN10-11 & -1.28 & -2.40 & -2.58 & -2.56 & -2.70 & -0.20 & -0.81 & -2.39 & -2.53 & -2.54 & -2.65 & -2.74 & -2.74 & -2.44 \\
\hline 32 & WO1 & -4.59 & -6.07 & -6.51 & -6.49 & -6.40 & -3.00 & -4.81 & -5.99 & -6.36 & -6.47 & -6.41 & -6.30 & -6.21 & -6.13 \\
\hline 40 & WO1-2 & -5.55 & -6.68 & -6.81 & -6.78 & -6.68 & -3.51 & -5.15 & -6.62 & -6.72 & -6.76 & -6.68 & -6.56 & -6.35 & -6.14 \\
\hline 60 & WO1-3 & -5.90 & -6.99 & -7.08 & -7.04 & -6.94 & -3.88 & -5.45 & -6.95 & -7.01 & -7.03 & -6.94 & -6.78 & -6.56 & -6.31 \\
\hline 85 & WO1-3 & -6.01 & -7.09 & -7.16 & -7.12 & -7.01 & -4.02 & -5.55 & -7.04 & -7.10 & -7.11 & -7.01 & -6.84 & -6.61 & -6.36 \\
\hline 120 & WO1 & -6.00 & -7.09 & -7.17 & -7.14 & -7.03 & -3.98 & -5.54 & -7.04 & -7.10 & -7.13 & -7.04 & -6.87 & -6.65 & -6.41 \\
\hline
\end{tabular}

the $V$-band. The $25 M_{\odot}$ non-rotating model is about one magnitude fainter than their rotating counterparts. Conversely, the non-rotating $32 M_{\odot}$ and $40 M_{\odot}$ models are significantly brighter in $U B V R I$ than the respective rotating models at the same initial mass, since the non-rotating models are significantly cooler (spectral type WN8) than the rotating models (spectral type WO1). Between $60 M_{\odot}$ and $85 M_{\odot}$, the non-rotating models are slightly fainter than the rotating ones, while the non-rotating $120 M_{\odot}$ model is about 2 mag brighter than the rotating $120 M_{\odot}$ model.

We present in Fig. 7 the bolometric corrections in the different bands as a function of initial mass. These can be employed to obtain the bolometric luminosity in the cases where observations were performed in a single band, and one has indications of 
Jose H. Groh et al.: Fundamental properties of core-collapse SN progenitors
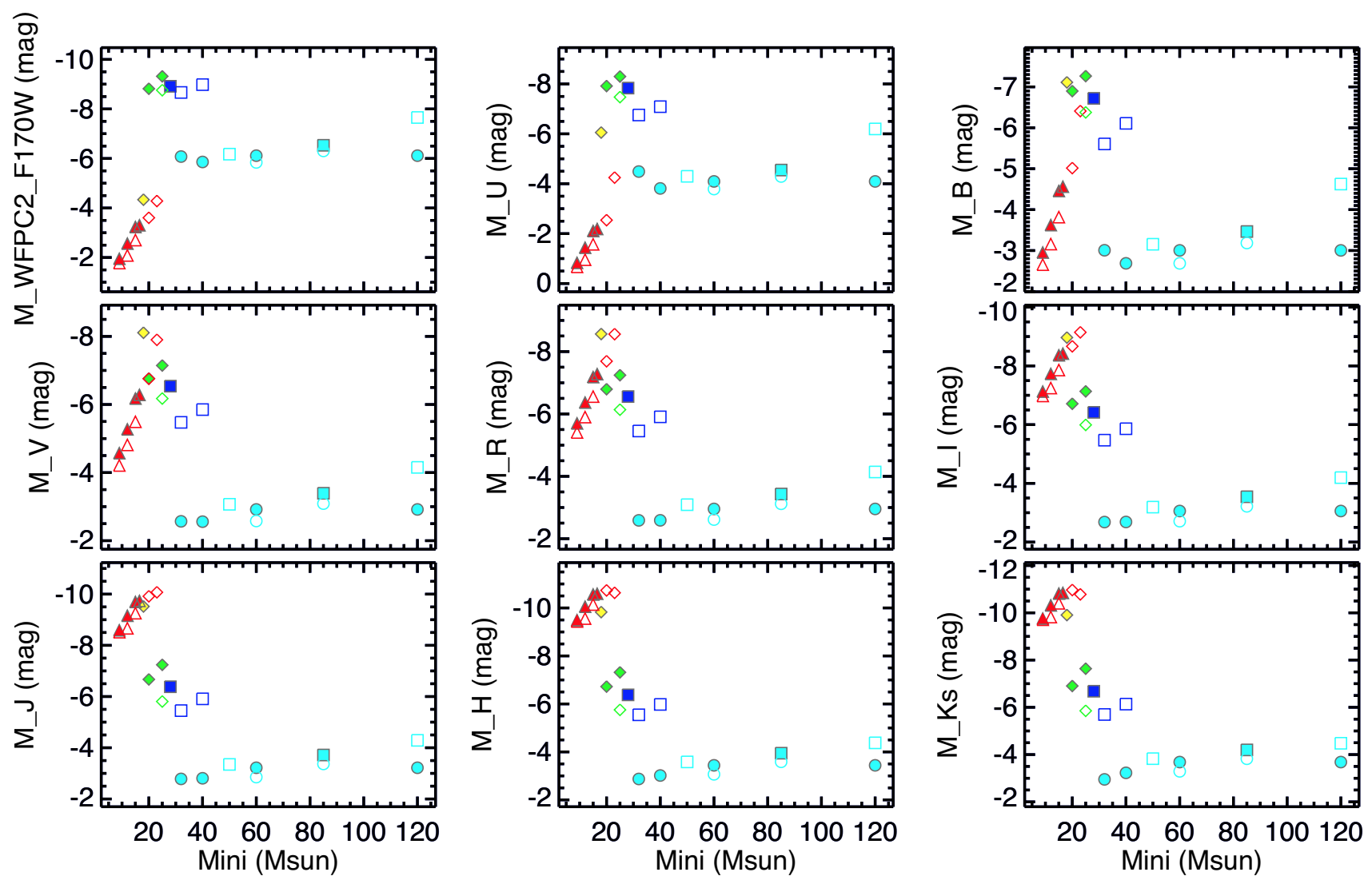

\begin{tabular}{|c|c|c|}
\hline $\begin{array}{c}\text { Progenitor Spectral Type } \\
\square \triangle \square \text { WO } \\
\square \square \text { WNL } \\
\diamond \diamond \text { LBV } \\
\diamond \diamond \mathrm{YHG} \\
\Delta \triangle \mathrm{RSG}\end{array}$ & $\begin{array}{l}\text { Supernova Type } \\
\bigcirc \bigcirc \text { Ic } \\
\square \square \square \square \text { Ib } \\
\diamond \diamond \diamond \diamond \text { IIL or Ilb } \\
\Delta \Delta \text { IIP }\end{array}$ & 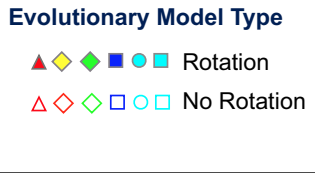 \\
\hline
\end{tabular}

Fig. 6. Absolute magnitude of SN progenitor models as a function of initial mass, in the WFPC2/F170W and UBVRIJHK filters (from top to bottom, left to right). We employ a three-dimensional scheme to label the symbols, according to the progenitor spectral type, SN type, and evolutionary model type (see lower panel). The progenitor spectral type is color coded, with cyan symbols corresponding to WOs, blue to WNL, green to LBVs, yellow to YHGs, and red to RSGs. The SN type is coded as the geometry of the symbol, with circles depicting progenitors of SN Ic, squares of SN Ib, diamonds of SN IIL/b, and triangles of SN IIP. The evolutionary model type is coded as open (without rotation) and filled (with rotation) symbols.

the nature of the progenitor. As one can see, the largest bolometric corrections (in modulus) are found for the WO stars, since they have most of their flux emitted outside the bandpass of the $U B V R I$ filters.

Figure 8 shows color-magnitude diagrams for the SN progenitor models discussed here. We can see that the progenitors from stars with higher initial mass $\left(M_{\text {ini }}>20 M_{\odot}\right)$ are blue and have negative $U-B, B-V$, and $V-I$ colors, since these are LBVs, WN8s, and WOs, all with relatively high values of $T_{\text {eff }}$. They are well separated in color from the SN progenitors from stars with $M_{\text {ini }}<20 M_{\odot}$, which are either RSGs and thus present red colors, or are YHGs, filling the gap between red and blue stars.

\section{Detectability of core-collapse SN progenitors}

\subsection{Progenitors of SN IIP}

Volume-limited SN surveys show that SN IIP should be the most common type of core-collapse SN, corresponding to about $48-55 \%$ of the total of core-collapse SNe (Smith et al. 2011a; Eldridge et al. 2013). Our results show that the progenitors of
SN IIP (triangles in Fig. 6) are the faintest progenitors in the $F 170 \mathrm{~W}$ and $U$ filters, becoming brighter in the red filters, and are the brightest ones in the $I J H K_{\mathrm{S}}$ filters. This is because they are RSGs, which have the peak of their flux emitted in the $I J H K_{\mathrm{S}}$ filters (Fig. 5). Starting from the $R$-band, even the faintest SN IIP progenitors have $M_{\text {bol }}<-5 \mathrm{mag}$, and thus should be the easiest to be detected in $R I J H K_{\mathrm{S}}$.

As found in previous studies (e.g., Humphreys \& McElroy 1984; Levesque et al. 2005; Davies et al. 2013), the absolute magnitudes of RSGs in a given filter significantly change as a function of temperature and luminosity. Since these are well correlated with the initial mass, one can use the absolute magnitudes of SN progenitors that were RSGs before the explosion to estimate their initial masses. Here we provide relationships between $M_{\text {ini }}$ and absolute magnitudes that should be applied to de-reddened photometry, keeping in mind that the reddening may be uncertain towards RSGs, which could affect the determination of the de-reddened photometry from the observations (Kochanek et al. 2012; Walmswell \& Eldridge 2012; Davies et al. 2013). Figure 9 shows the variation of the absolute magnitude in different filters of the HST/WFPC2 system, which is commonly employed in studies of SN progenitors, as a function 

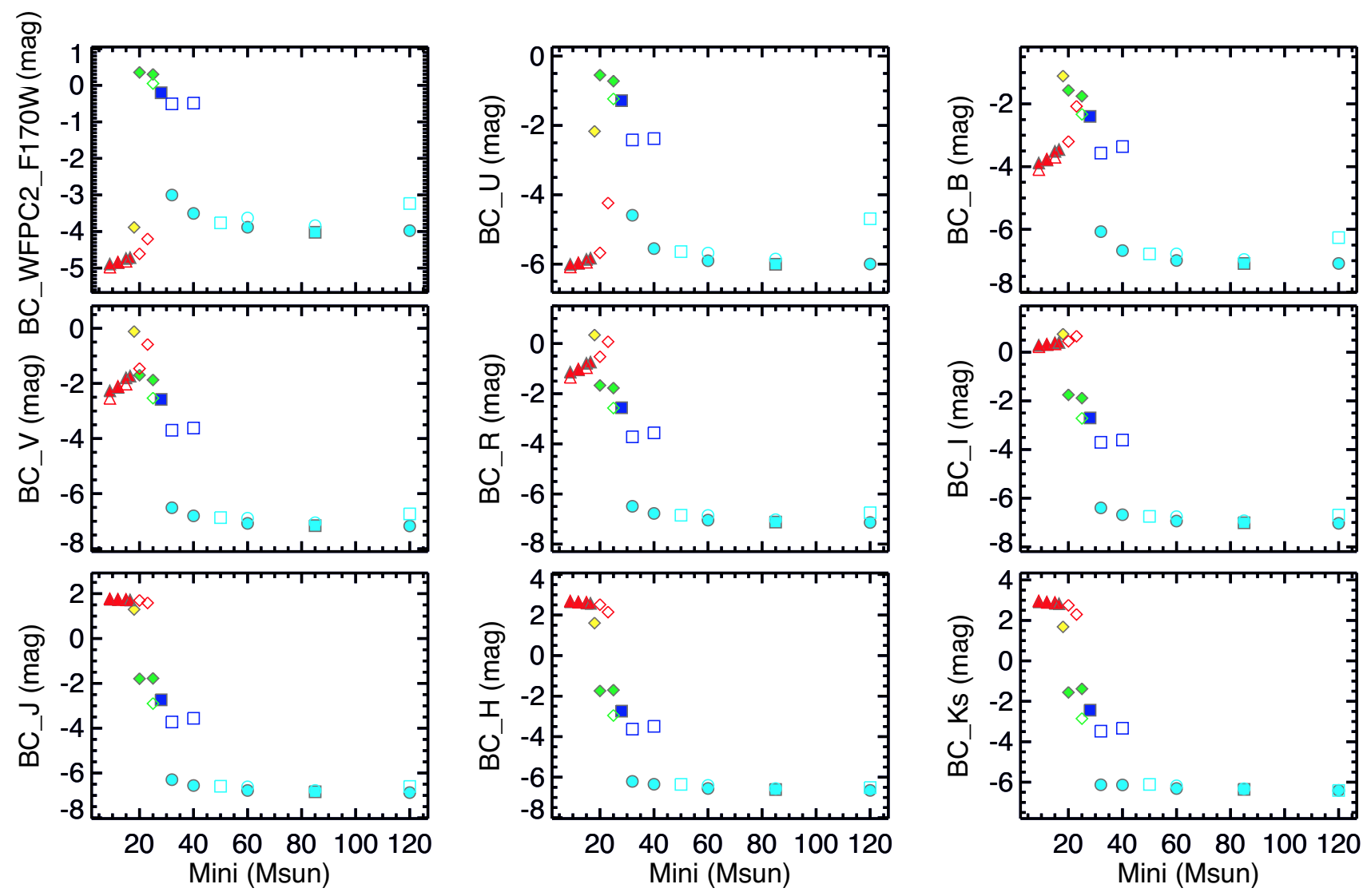

\begin{tabular}{|ccc|}
\hline Progenitor Spectral Type & Supernova Type & Evolutionary Model Type \\
$0 \square, \square$ WO & $\circ \circ$ Ic & $\Delta \diamond \diamond \square \circ \square$ Rotation \\
$\square \square$ WNL & $\square \square \square \square$ Ib & $\Delta \diamond \diamond \square \circ \square$ No Rotation \\
$\diamond \diamond$ LBV & $\diamond \diamond \diamond \diamond \mathrm{IIL}$ or Ilb & \\
$\diamond$ YHG & $\Delta \Delta$ IIP & \\
$\Delta \Delta$ RSG & & \\
\hline
\end{tabular}

Fig. 7. Similar to Fig. 6, but showing the bolometric corrections as a function of initial mass of the SN progenitors.
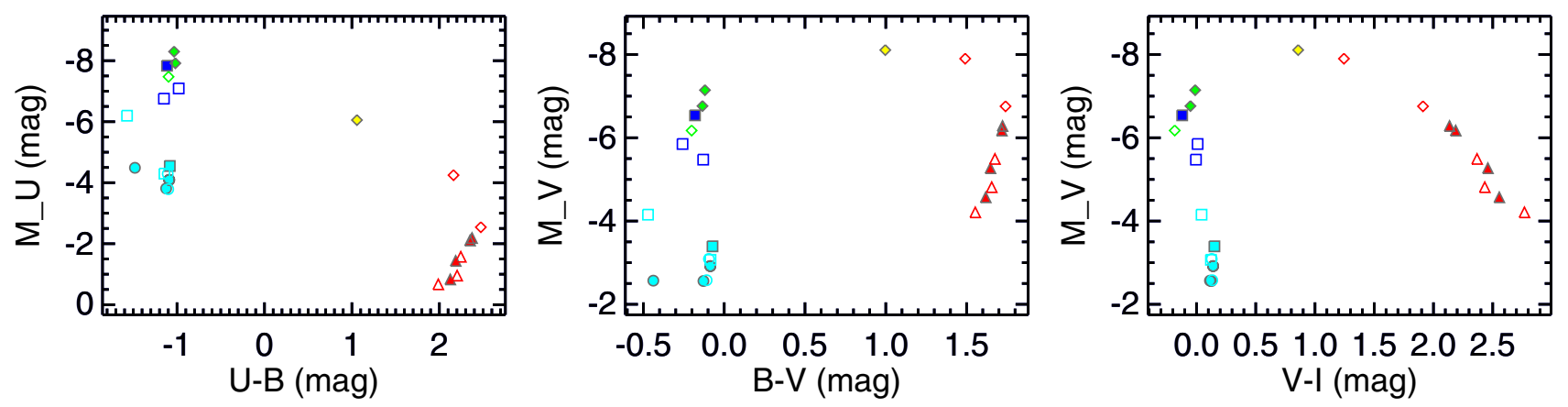

Fig. 8. From left to right, color-magnitude diagrams $M_{U}$ vs. $(U-B), M_{V}$ vs. $(B-V)$, and $M_{V}$ vs. $(V-I)$. Symbols have the same meaning as in Figs. 6 and 7.

of initial mass for the models that are RSGs at their end stage. In most filters, one can clearly devise a linear variation of the absolute magnitude as a function of initial mass, both for rotating and non-rotating models. We notice, however, that the absolute magnitude of rotating models seem to present a plateau and level off above $15 M_{\odot}$. Therefore, we computed the following least-square linear fits for rotating models, valid in the range $9 M_{\odot}<M_{\text {ini }}<15 M_{\odot}$ :

$$
\begin{aligned}
& M_{\text {ini }}\left(M_{\odot}\right)=-3.977-3.903 M_{F 450 W} \\
& M_{\text {ini }}\left(M_{\odot}\right)=-10.165-3.753 M_{F 606 W} \\
& M_{\text {ini }}\left(M_{\odot}\right)=-25.108-4.827 M_{F 814 W}
\end{aligned}
$$

while for non-rotating models we found (valid in the range $\left.9 M_{\odot}<M_{\text {ini }}\left(M_{\odot}\right)<23 M_{\odot}\right)$ :

$$
\begin{gathered}
M_{\mathrm{ini}}\left(M_{\odot}\right)=-4.277-4.480 M_{F 450 W} \\
M_{\mathrm{ini}}\left(M_{\odot}\right)=-11.566-4.346 M_{F 606 W} \\
M_{\mathrm{ini}}\left(M_{\odot}\right)=-32.822-6.129 M_{F 814 W} .
\end{gathered}
$$

It is readily apparent that rotating and non-rotating models follow different relationships. For the same mass, we find that rotating models are systematically brighter than non-rotating models, in all filters. This is due to the rotating models having higher $T_{\text {eff }}$ and $L_{\star}$ than non-rotating models at the same mass. The higher 
Jose H. Groh et al.: Fundamental properties of core-collapse SN progenitors
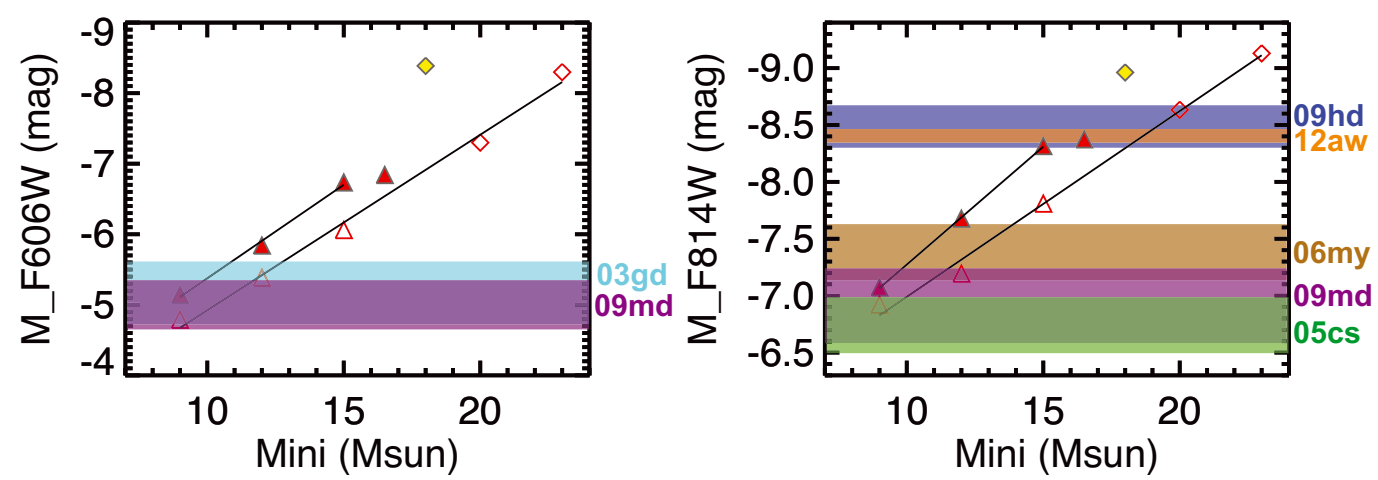

Fig. 9. Absolute magnitudes in the HST/WFPC $F 606 W$ and $F 814 W$ filters as a function of initial mass for RSGs at the pre-SN stage. Symbols are as in Fig. 6. Least-squares linear fits for both models (see text) are also shown. The colored bands represent the absolute magnitude \pm 1 sigma of observed RSGs that underwent core-collapse SN.

$L_{\star}$ in rotating models is caused by the larger He cores in these models compared to non-rotating models.

Since it is impossible to distinguish, based on photometry alone, whether a SN progenitor comes from an initially rotating or non (slowly) rotating star, this implies a degeneracy between the absolute magnitude at the pre-SN stage and the initial rotation and initial mass of SN progenitors that were RSGs. Because of this degeneracy, there is an uncertainty in the predicted initial mass of the SN progenitor. When analyzing the progenitor of SN2012aw, Van Dyk et al. (2012) reached a similar conclusion when comparing their results based on rotating models from Ekström et al. (2012) with those from Fraser et al. (2012), which in turn had employed non-rotating STARS models (Eldridge et al. 2008).

Once the absolute magnitude in a given filter of a SN progenitor is known, its mass can be estimated by employing Eqs. (4)-(9), assuming it is a RSG. These relationships assume that stars have their terminus in the HR diagram with $T_{\text {eff }}$ and $L_{\star}$ as predicted by our evolutionary models. Under this assumption, we are thus able to constrain the mass of observed SN progenitors in a homogeneous way, as shown in Fig. 9. Table 6 presents the assumed distance, reddening, absolute magnitudes, and mass estimate of SN progenitors collected from the literature, together with our estimates. We consider here only progenitors that are in galaxies consistent with solar metallicity, with oxygen abundance (by number) of $\log (\mathrm{O} / \mathrm{H})+12 \simeq 8.4-8.7$.

In the cases where a progenitor has been found, such as $\mathrm{SN}$ 2003gd (Smartt et al. 2009; Maund et al. 2013), SN 2005cs (Maund et al. 2005a; Li et al. 2006; Eldridge et al. 2007; Smartt et al. 2009), SN 2006my (Maund et al. 2013), SN 2009hd (EliasRosa et al. 2011), SN 2009md (Fraser et al. 2011), and 2012aw (Fraser et al. 2012; Van Dyk et al. 2012), our estimates for the non-rotating and rotating models bracket the values derived by the Smartt group well, which employ non-rotating STARS models. In the cases where only an upper limit to the absolute magnitude of the SN progenitor is available, we derive in general lower values for the upper initial mass limit, both for non-rotating and rotating models. This results from the fact that we assume that RSGs at their end phase have $T_{\text {eff }}$ and $L_{\star}$ as predicted by our evolutionary models at the end of core-C burning (Table 2). Conversely, while the Smartt group has also assumed that RSGs most likely explode at the end of their evolution, they adopted a conservative approach to compute the progenitor upper mass limit, taking into account that the star could actually have exploded at any point after the end of core-He burning. Also, because rotating models are brighter than non-rotating models of the same mass, our upper mass limit is lower for the rotating models.

\subsection{Progenitors of $\mathrm{SN} \mathrm{Ibc}$}

With our database of SN Ibc progenitor spectra and absolute magnitudes, we are able to assess their detectability with much more realism than before. Previous works employed empirical calibrations of absolute magnitudes and bolometric corrections (Yoon et al. 2012; Maund \& Smartt 2005; Maund et al. 2005b; Crockett et al. 2007). More recently, Eldridge et al. (2013) employed atmospheric models of WR stars from the Potsdam group (Hamann et al. 2006; Sander et al. 2012) that were computed for a range of physical parameters, but not self-consistently with the evolutionary calculations. Here, our quantities are calculated self-consistently for each progenitor based on our combined stellar evolution and atmospheric modeling. However, we recall here the caveats associated with classifying the SN type based on the chemical composition of the ejecta, and the challenges of distinguishing between $\mathrm{SN}$ Ib and Ic based on the He abundance (e.g. Dessart et al. 2011). The following discussion assumes that SN Ib have more than $0.6 M_{\odot}$ of He in their ejecta, while quantities smaller than this would lead to SN Ic.

So far, no WR star has been directly detected as a SN progenitor (Smartt 2009; Eldridge et al. 2013). Since single-star massive evolution models predict WRs as SN progenitors, the yet non-detection may sound surprising. It has been proposed that binary evolution could be absolutely needed to explain the nondetections (Smartt 2009; Eldridge et al. 2013). However, using empirical bolometric corrections based on WR models from the Potsdam group, Yoon et al. (2012) suggested that at their end stage, single WR stars have faint absolute magnitudes in the optical bands, since they are extremely hot $\left(\log \left(T_{\text {eff }} / \mathrm{K}\right)>5.0\right)$. This would make them undetectable in the optical pre-explosion images of SN Ibc progenitors.

Here, we showed more specifically that the progenitors of SN Ibc are WO 1-3, WN7-8, WN10-11 stars, and computed their absolute magnitudes and bolometric corrections. We find that, contrary to RSGs, the absolute magnitudes of the WOs that are SN progenitors do not show a clear scaling with initial mass. This happens because the models with higher $M_{\text {ini }}$ finish at higher luminosities but also at hotter temperatures. This increase in $T_{\text {eff }}$ causes more flux to be emitted outside the optical and near-infrared bands, which roughly compensates the increase in flux caused by the higher luminosity. Therefore, when photometric observations of $\mathrm{SN}$ Ibc detect their progenitors, our models 


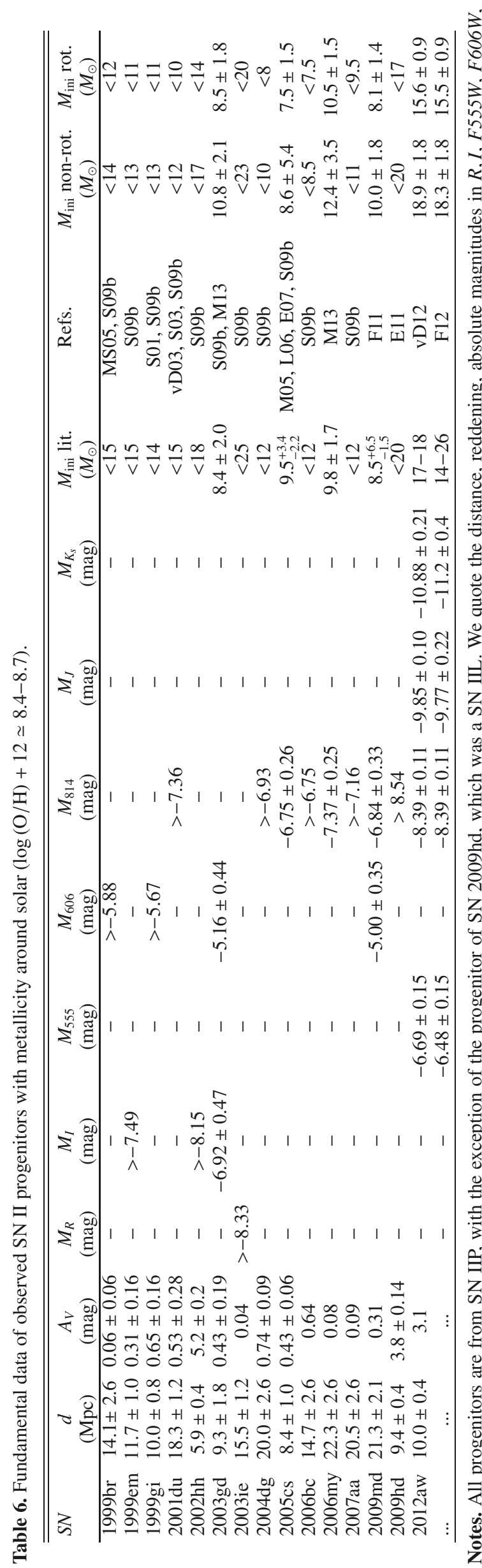

हैं ป

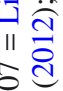

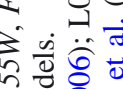

记 엉

-

긴

을

च

on 8 के

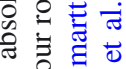

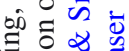

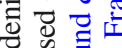

of $\sum^{2} \frac{N}{11}$

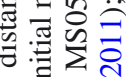

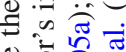

ํ. 융

헌

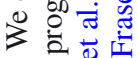

뵤혜

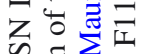

\%.

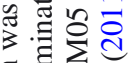

可的

3 워

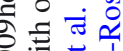

잉

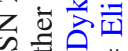

पै

호거

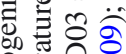

뉸용

ô

प्ट

흥 छ

일 च

跤 II

छ छี

के $\|$ क

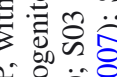

刍 웋웡

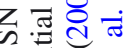

짐워

综

औे

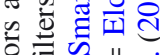

结

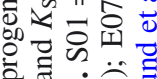

云额

《र

ब这

迹
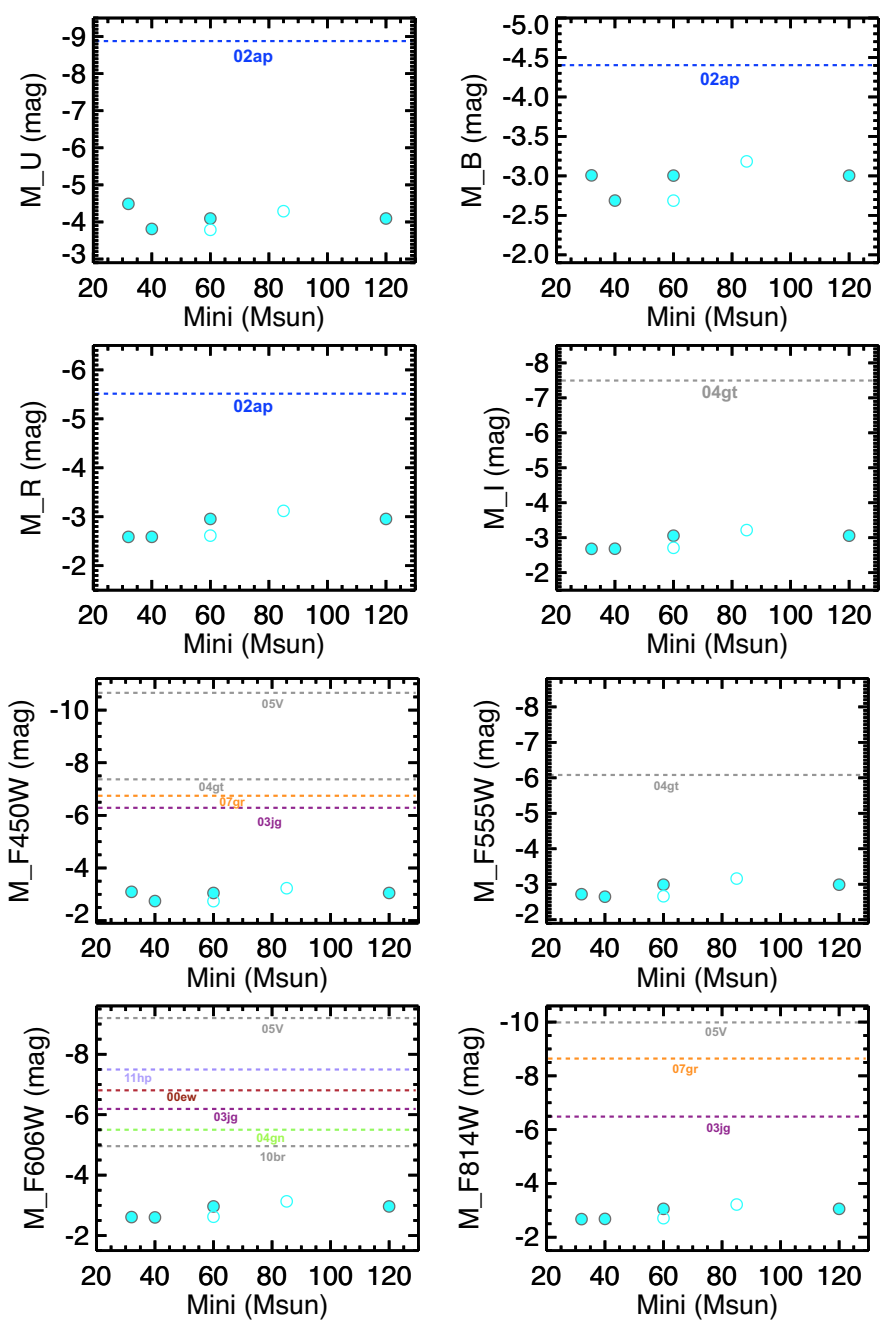

Fig. 10. Absolute magnitude of our models that are SN Ic progenitors (open and filled cyan circles) compared to observed upper magnitude limit for different SNe Ic (colored horizontal dashed lines followed by shortened SN label) and SN Ibc (gray horizontal dashed lines). Open (filled) symbols correspond to non-rotating (rotating) models. From top to bottom, we show the comparison between model and observations in the UBRI and HST/WFPC2 F450W, F555W, F606W, and F814W filters. Note that all models lie below the detectability limit of all SN Ic and Ibc.

predict that it will be challenging to assign a stellar mass with similar precision as has been done for RSGs.

Let us investigate whether our SN Ib and SN Ic model progenitors would be detectable in the available observational data. We adopt the observed magnitude limits compiled by Eldridge et al. (2013) of progenitors of 6 SN Ic, 3 SN Ib, and 3 SN Ibc. In this context, a SN Ibc classification means that the distinction between a Ib or Ic classification is unclear. Figure 10 presents the observed detection limits in the absolute magnitude of SN Ic progenitors compared to the predictions of our models in different filters. We find that all SN Ic model progenitors would be undetectable in the available pre-explosion images. Included in Fig. 10 are also SN Ibc progenitors, showing that if these were SN Ic, their progenitors would be undetectable too. In the case of the SN Ic progenitor with the deepest magnitude limit available $\left(M_{B} \simeq-4.4 \mathrm{mag}, \mathrm{SN} 2002 \mathrm{ap}\right)$, our models predict that even the brightest SN Ic progenitor would still be $1.5 \mathrm{mag}$ fainter than the detection limit. Our conclusion is that SN Ic progenitors from single stars can easily evade detection in pre-explosion images, 
since they have spectral type WO and are faint in the optical/NIR bands. This is because they are extremely hot at their endpoints, in agreement with the suggestion from Yoon et al. (2012). Thus, our models of single stars are in agreement with the lack of detection of SN Ib and Ic progenitors at the current magnitude limit of the observations.

Our results are in contrast with the findings of Eldridge et al. (2013), who find a $13 \%$ of probability of non-detection of the progenitors of SN Ib and Ic based on single star evolution models, and $12 \%$ non-detection probability based on binary evolution models. The reason for this discrepancy is that we assume, as in Yoon et al. (2012), that stars will explode only at the end point of the evolution predicted by stellar evolution models. Eldridge et al. (2013), on the contrary, assumes that the stars can explode anytime after the end of core-helium burning. Our models predict that there is significant increase in the effective temperature of the star after the end of core-helium burning, so assuming an explosion before the end point of the evolution would underestimate the temperature of the progenitor, and overestimate its absolute magnitude. Therefore, our models predict much fainter progenitors than those from Eldridge et al. (2013).

One may wonder what would be the maximum distance that a SN Ic progenitor should be to be still detectable according to our predicted absolute magnitudes. This obviously depends on the magnitude limits and the amount of reddening towards the progenitor. For typical detection limits $(m=24.5)$ and low amounts of extinction ( $0.3 \mathrm{mag}$ in a given band), this implies a maximum distance to SN Ic progenitors of $2.7 \mathrm{Mpc}$ for detection. For a SN 2002ap-like detection limit $\left(m_{B}=26.0 \mathrm{mag}\right.$, Crockett et al. 2007), the maximum distance would be $5.5 \mathrm{Mpc}$. Obviously, these are upper limits that would decrease if the progenitor is seen under large extinction. We predict that the limiting magnitudes in the observations should be at least 2 mag fainter than the current best limit in the $B$ band to detect single star progenitors of SN Ic at distances of 10-20 Mpc.

The absolute magnitude of SN Ib progenitors from our models and the detection limits derived from observations are shown in Fig. 11. In all filters, we see that the three observed magnitude limits of $\mathrm{SN} \mathrm{Ib}$ progenitors are brighter than the absolute magnitude predicted by our models of SN Ib progenitors. Therefore, according to our models, the progenitors of SN 2001B, SN 2011am and SN 2012au should not have been detected, in agreement with the observations.

The magnitude limits of the progenitors of the SN Ibc SN 2004 gt and SN 2010br are also show in Fig. 11. As mentioned above, these are $\mathrm{SNe}$ where the classification is uncertain between $\mathrm{Ib}$ and Ic. Figure 11 reveals that the progenitors of SN 2004gt and SN 2010br could have been detected in pre-explosion images if they were progenitors of SN Ib. Since our models predict some of the $\mathrm{SN}$ Ib progenitors to be much brighter than the SN Ic progenitors, to determine the detectability of the progenitor it is thus of crucial importance to constrain the SN type between Ib and Ic. To asses the detectability of the progenitors of SN 2004gt and SN 2010br, we compute the probability that a SN progenitor would not be detected in available pre-explosion images. For this purpose, we assume that the progenitor is randomly drawn from the mass range that produces a SN Ib or Ic, a Salpeter IMF, the initial mass ranges that generates SN Ibc from Georgy et al. (2012), and that half of the stars evolve from rotating stars, while the other half evolve from slow-rotating stars (thus described by our non-rotating models). We also linearly interpolate the synthetic model absolute magnitudes as a function of initial mass. We obtain that the progenitors of SN2004gt and SN 2010br have a probability of non-detection
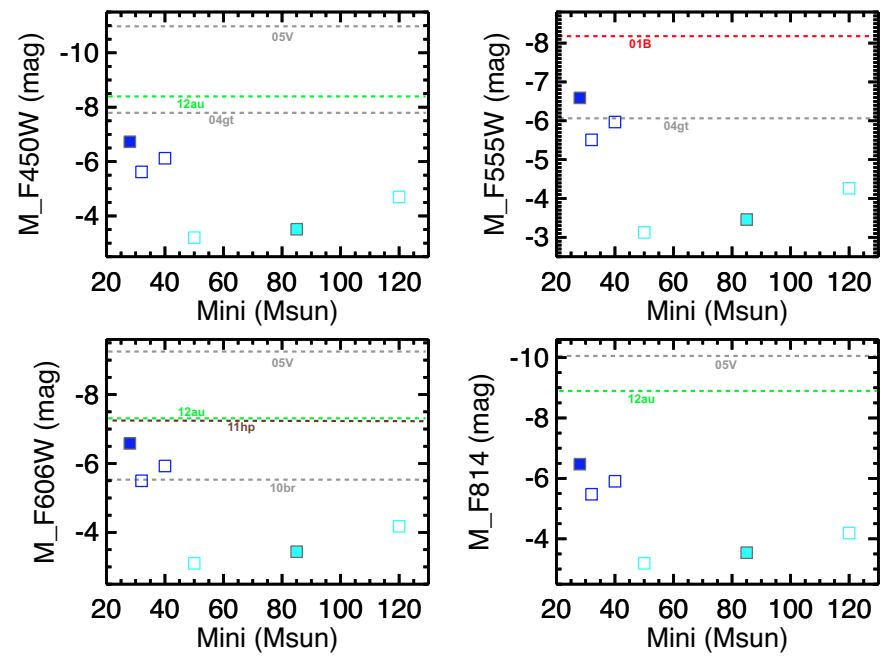

Fig. 11. Similar to Fig. 10, but for SN Ib progenitors.

in the pre-explosion images of $87 \%$ and $62 \%$, respectively. As in the case of SN 2002ap, our non-detection probabilities are higher than those computed by Eldridge et al. (2013), and the reasons are the same as for SN 2002ap.

All in all, we see that the current non-detection of progenitors of SN Ib and SN Ic in pre-explosion images cannot be used as an argument to discard single stars as the progenitors of these types of SNe. The present discussion also shows that to really discard the single star scenarios for a given progenitor, no detection should be made even when the limiting magnitudes in the observations would be at least 2 mag fainter than the current best limit in the B band. Therefore, if our models are correct, binarity is not necessarily needed to explain the non-detections.

\section{WO stars as progenitors of SN Ibc}

Our results present a paradigm shift in the sense that for the most massive models, we find a WO spectral type before core collapse, and not a WC spectral type as has been widely assumed based on the chemical composition of the progenitor (Georgy et al. 2009, 2012; Eldridge et al. 2008). But is this shift in paradigm supported by observations? Based on the spectrum, our models indicate that the WO phase is a short evolutionary stage at the end, with duration of a few 10000 years (Groh et al. 2013, in prep.). The WC phase, on the other hand, corresponds to where the star spends most of its He burning phase, and thus has a relatively long duration. Therefore, one would expect that WC stars are a large fraction of the total number of WR stars, while WO stars should be extremely rare. Indeed, out of a population of 576 Galactic WR stars ${ }^{3}$, only four WO stars are known in the Galaxy (one of which is in a binary system). From a zeroth-order calculation of the expected ratio WO/WR based on our theoretical models and the predicted timescales (Groh et al. 2013 , in prep.), one would expect 0.5 to $2 \%$ of the total WRs to be WOs. This is in line with the observed rarity of WO stars.

We plot the location of Galactic WO and WC stars in the HR diagram in Fig. 12, assuming the parameters determined by Sander et al. (2012) and restraining the analysis to the objects with known distances. For simplicity, we plot their $T_{\star}$ (computed at a Rosseland optical depth of 20) as corresponding to the

3 According to the latest compilation of WR stars by P. Crowther, v1.5, Feb. 2013; http://pacrowther. staff. shef .ac.uk/WRcat 


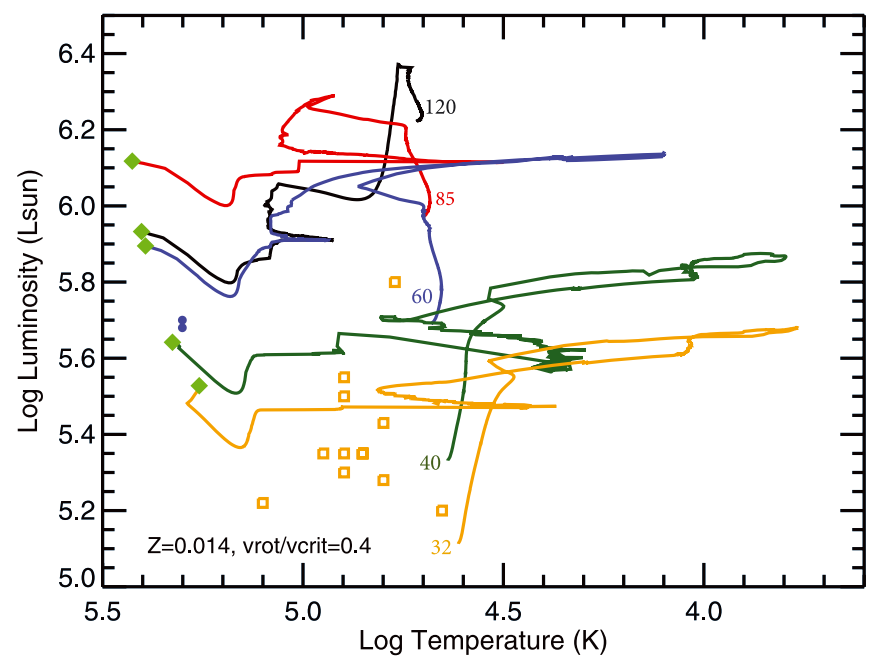

Fig. 12. Evolutionary tracks of rotating models from Ekström et al. (2012), as those in Fig. 1, with the endpoints indicated by green diamonds. The position of Galactic WO stars (filled blue circles) and WC stars with known distances (open orange squares) are overplotted for comparison.

values of $T_{\star}$ from our stellar evolution models. As discussed by Sander et al. (2012), this assumption could lead to uncertainties of $0.1-0.2$ dex in $T_{\star}$, which would not affect our conclusions below. We can immediately notice in Fig. 12 that the two observed Galactic WO stars, WR 102 and 142 (filled blue circles), lie extremely close to the endpoints of the rotating $40 M_{\odot}$ track (and to the non-rotating $60 M_{\odot}$ track, not shown in Fig. 12; see Fig. 1). This would mean that their initial masses were around 40-60 $M_{\odot}$, while errors in the $L_{\star}$ and/or $T_{\star}$ determination would shift the initial mass between $32-120 M_{\odot}$, but still putting them close to the endpoint of the evolution. Therefore, the position of observed Galactic WO stars is consistent with our model results, which show that WO stars are the immediate stage before core collapse. In addition, the chemical surface abundances predicted by our models are similar to those derived for WOs by Sander et al. (2012), with a high $\mathrm{C}$ and $\mathrm{O}$ content. We can say that our rotating models are corroborated by observations in the range 32-60 $M_{\odot}$. Sander et al. (2012) reached a similar conclusion by comparing their observations with the Meynet \& Maeder (2003) non-rotating evolutionary tracks.

No WO star with $\log L_{\star} / L_{\odot}>5.7$ has been detected in the Galaxy. In the HR diagram, these more luminous WOs would be in a place close to the endstage of the tracks with $M_{\text {ini }}>60 M_{\odot}$. One may then rightfully wonder whether stars born more massive than $60 M_{\odot}$ indeed end their lives as WO stars. We point out that the non-detection of WOs could be due to the short timescale related to this phase (5000-10000 years), which would make the detection quite unlikely. This would be combined with the scarcity of stars above $M_{\text {ini }}>60 M_{\odot}$, as for a Salpeter initial mass function, one would expect approximately twice as few stars between $60-120 M_{\odot}$ than in the 32-60 $M_{\odot}$ range. Finally, as shown in Figs. 2 and 3, the detection of WO stars may be hampered by the possibly weak lined-spectrum that could be characteristic of these stars, depending on their $\dot{M}$. Since most WR stars are identified based on narrow-band surveys, one would find challenging to detect weak-lined WO stars in narrow-band surveys using the C IV $\lambda 5808$ line, for instance. Our conclusion is that the current non-detection of WO stars with $\log L_{\star} / L_{\odot}>5.7$ is expected given their rarity.
An additional support for WO stars being the endstage of single massive stars in the range 32-120 $M_{\odot}$ comes from the fact that the observed WC stars with known distances (orange squares in Fig. 12) are located significantly far from the endpoint of the stellar evolutionary tracks. However, due to the fact that WC stars may have inflated radii (Gräfener et al. 2012), it might be that, once corrected for this inflation effect, the stars would actually populate the region near the end points of the evolutionary tracks (for recent discussions see Sander et al. 2012 and Georgy et al. 2012). We do not think that this effect could shift sufficiently to the blue the observed positions of the WC stars. This is because at the endmost stages, $T_{\star}>150000$, which is the characteristic temperature of the opacity peak due to Fe. As shown by Gräfener et al. (2012), one needs $T_{\star}<150000$ in order for inflation to occur, as the Fe opacity bump region needs to be within the envelope of the star, and not in the stellar wind as it occurs when $T_{\star}>150000$.

Our models also show that WO stars arise naturally at solar metallicity at the end stage of the evolution. Therefore, lower metallicities are not necessary to produce WO stars as SN Ibc progenitors. Also, WO stars occur both in rotating and nonrotating models, with the difference being that rotation diminishes the minimum initial mass to produce WO stars from $60 M_{\odot}$ to $32 M_{\odot}$. As such, WO stars do not come necessarily from only rapid rotators.

As for gamma-ray bursts (GRB) progenitors, we note that GRBs seem to occur, albeit at lower frequency, even at metallicities around the solar values (Levesque et al. 2010). Georgy et al. (2012) showed that 40 and $60 M_{\odot}$ rotating models discussed here have a large amount of core angular momentum, which seems to make them favorable for GRB production. If this turns out to indeed occur, our results here indicate that the GRB progenitors at solar metallicity have a WO spectral type.

\section{When LBVs are SN progenitors and their $\mathrm{SN}$ types}

From a theoretical perspective, LBVs have been only recently linked to the pre-SN stage of massive stars (Groh et al. 2013). In that paper, we showed that stars with $M_{\text {ini }}=20-25 M_{\odot}$ end their lives with properties similar to LBVs, such as the spectral morphology, the proximity to the Eddington limit, and the chemical abundances.

In addition, as can be inferred from their values of $T_{\text {eff }}$ $(\sim 20000 \mathrm{~K})$, we note here that our $M_{\text {ini }}=20-25 M_{\odot}$ rotating models flirt with the so-called bistability limit of line-driven winds. We refer the reader to Pauldrach \& Puls (1990), Lamers et al. (1995), and Vink et al. (1999) for detailed discussions. In short, the bistability is related to a change in the optical depth of the Lyman continuum, which in turn changes the ionization structure of metals in the wind, in particular Fe. This occurs at $T_{\text {eff }} \sim 21000 \mathrm{~K}$. As a consequence, the amount of line driving is affected, ultimately changing $\dot{M}$ and $v_{\infty}$. Classical LBVs such as AG Car (Vink \& de Koter 2002; Groh et al. 2009b, 2011) and P Cygni (Pauldrach \& Puls 1990; Najarro et al. 1997; Najarro 2001) are well known for being close to the bistability limit. In the context of our models, being close to the bistability limit may produce an inhomogeneous circumstellar medium (CSM) in the vicinity of the progenitor (Moriya et al. 2013). It is interesting to note that the inference of wind variability from the radio lightcurve was the first way to link LBVs as SN progenitors (Kotak \& Vink 2006), and theoretical work has shown that LBVs produce inhomogeneous CSM when they cross the bistability (Groh \& Vink 2011). 


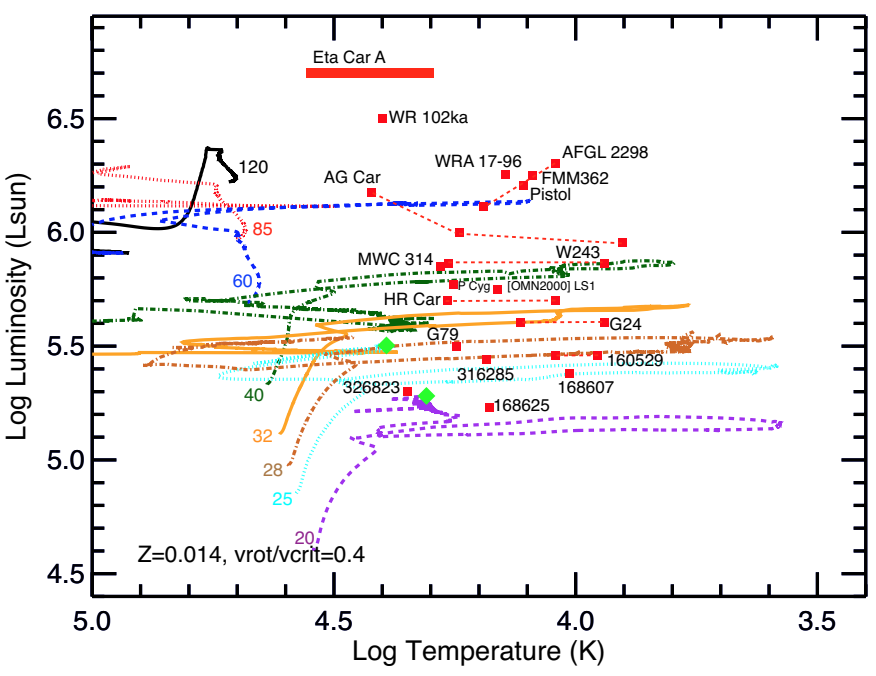

Fig. 13. Position of Galactic LBV stars (red squares) in the HR diagram. The sources of the stellar parameters of the LBVs are as follows: Eta Car A (Groh et al. 2012; Hillier et al. 2001), WR 102ka (Barniske et al. 2008), AG Car Groh et al. (2009b; 2011; Groh et al., in prep.), AFGL 2298 (Clark et al. 2009a), FMM 362 and Pistol star (Najarro et al. 2009), Wray 17-96 (Egan et al. 2002), G24.73+0.69 (Clark et al. 2009a), [OMN2000] LS1 (Clark et al. 2009b), HD 316285 (Hillier et al. 1998), HD 160529, HD 168625 and HD 168607 (van Genderen 2001), P Cygni (Najarro 2001; 2011, priv. comm.), HD 326823 (Marcolino et al. 2007), G79.29+0.46 (Trams et al. 1999), and W243 (Ritchie et al. 2009). For comparison, we show evolutionary tracks of rotating models from Ekström et al. (2012), as those in Fig. 1, with the endpoints indicated by green diamonds.

Are all LBVs at the end stage of massive star evolution, or are some LBVs in a transitional stage towards the WR phase? This is a fair question that should be posed, in particular if one's goal is to obtain the evolutionary status of observed LBVs. Here we tackle this question from a theoretical perspective. For that purpose, we plot in Fig. 13 the position of the Galactic LBVs and LBV candidates in the HR diagram, together with our evolutionary tracks for rotating stars. We immediately note in Fig. 13 that LBVs are distributed over a range of luminosities (see also van Genderen 2001; Smith et al. 2004; Clark et al. 2005, 2009a, 2012b; Vink 2012). If LBVs arise from the evolution of single stars, the spread in luminosity means that they come from a range of initial masses. According to our models, LBVs would arise from stars with $M_{\text {ini }} \geq 20 M_{\odot}$. We predict that only the 20-25 $M_{\odot}$ tracks have their pre-SN stage as LBVs, and all LBVs that arise from stars with $M_{\text {ini }}>25 M_{\odot}$ will further evolve to the WR stage.

It is important to point out that, contrary to previous studies (Smith et al. 2004), there is no clear observational evidence of separation between low- and high-luminosity LBVs. This is a result of our updated compilation of the fundamental parameters of LBVs, in which most LBVs have been analyzed with nonLTE radiative transfer atmospheric codes (mainly CMFGEN). A similar conclusion can also be drawn from the parameters quoted in Clark et al. (2005, 2009a, 2012b).

Obtaining the precise evolutionary status of observed LBVs is, however, a challenging task, because one has to rely on matching $L_{\star}$ and $T_{\star}$ of each individual star. Both quantities vary wildly in LBVs, on short timescales as a result of S-Dor type instability (changes in $R_{\star}$ and $L_{\star}$ without significant changes in $\dot{M}$ see Groh et al. 2009b) and, more rarely, Giant
Eruptions (ejections of several solar masses in a short outburst with increase in $L_{\star}$, see Humphreys \& Davidson 1994). Both phenomena are not included in our models. In addition, LBVs may suffer from inflated radii (Gräfener et al. 2012). Taking the model predictions at face value, one would expect that LBVs with $\log L_{\star}<5.3$ would be close to the end of their evolution, while those with $\log L_{\star}>5.6$ would evolve to the WR phase. Given the uncertainties, LBVs with $5.3<\log L_{\star}<5.6$ could either come from $M_{\text {ini }}>25 M_{\odot}$ and be at an intermediate stage of their evolution, or come from $M_{\text {ini }}=20-25 M_{\odot}$ and be close to core collapse. It is worth noting that most LBVs have lower temperatures than the final stage of our 20-25 $M_{\odot}$ models. This could point towards the observed LBVs having inflated radii, or having long S-Dor variability cycles, or not being at the endmost stage of their evolution. In addition, it could be also a result of the models not being tailored to match a particular star, as one could fine tune the rotation rate and mass lost at the RSG stage to bring the endpoints to lower temperatures.

In the above picture, one has to take the uncertain distance determinations for most LBVs into account, meaning that significant errors in $L_{\star}$ could exist. As an example, the LBV AG Car has had distance determinations in the range 2-6 kpc (Humphreys et al. 1989). A larger distance has been preferred, but it is mainly anchored on the kinematical determination based on the Galactic rotation curve (Stahl et al. 2001; Groh et al. 2009b). If AG Car were at a much closer distance of $\sim 2-3 \mathrm{kpc}$, its $L_{\star}$ would be much lower and fall within the range where AG Car would be an immediate SN progenitor. Stellar parameters of LBVs can nowadays be determined with reasonable accuracy with the advent of non-LTE radiative transfer codes such as CMFGEN. The speculative scenario above illustrates that constraining the fate of observed Galactic LBVs relies on more precise distance determinations, which may become available with the GAIA mission.

From a theoretical perspective, let us discuss the types of SN that would arise from stars that explode as LBVs according to our models, having in mind the aforementioned caveats associated with linking the chemical abundances of progenitors with SN types. In Groh et al. (2013), we showed that the 20-25 $M_{\odot}$ models have a very small amount of $\mathrm{H}\left(\sim 0.02 M_{\odot}\right)$ left in their envelopes. This could point towards a $\mathrm{SN}$ IIb, meaning that LBVs could be the progenitors of SN IIb, such as SN 2008ax (Groh et al. 2013). However, until more firm constraints are put on the amount of $\mathrm{H}$ that characterizes the different types of SN II, we cannot rule out a type IIL. Interestingly, from an observational point of view, the original proposition that LBVs could be $\mathrm{SN}$ progenitors come from a study of transitional SNe and the effects of wind variability in their radio lightcurves (Kotak \& Vink 2006). These were SN 2001ig (type II that evolved to Ib; Phillips et al. 2001; Clocchiatti 2002; Filippenko \& Chornock 2002) and SN 2003bg (type Ic, later evolving to type II; Filippenko \& Chornock 2003; Hamuy et al. 2003). Therefore, there is observational support to our theoretical prediction that LBVs with relatively low luminosity are the progenitors of some SN IIb and other intermediate SN types between II and Ib.

Let us hypothesize what would happen in the case that a giant outburst occurred in the few years before the SN explosion of the 20-25 $M_{\odot}$ models. This phenomenon is not included in our stellar evolution models, since the physical mechanism is unknown. As we discussed in Groh et al. (2013), the progenitors of 20-25 $M_{\odot}$ rotating models, being LBVs and crossing the "Yellow Void" in the HR diagram, could experience episodic mass loss. This would give rise to a dense circumstellar medium (CSM) characterized by relatively low velocities. The interaction 
of the SN blast wave with the CSM could then create a SN with narrow lines. The SN type would in principle depend on the amount of $\mathrm{H}$ in the ejecta, which in the case of our models is 0.02 and $0.00 M_{\odot}$ for the 20 and $25 M_{\odot}$ models, respectively. In the case of the rotating $20 M_{\odot}$ model, a giant outburst in the few years before the SN could produce a CSM with a range of relative $\mathrm{H}$ abundance values, depending on the amount of mass ejected. A SN IIn would arise if the amount of mass ejected in the outburst is small (up to a few tenths of a solar mass). In this case, $\mathrm{H}$ would still be sufficiently abundant in the ejecta to produce the typical $\mathrm{H}$ narrow lines seen in SNe IIn. For a more violent giant outburst, one would think that the fractional abundance of $\mathrm{H}$ in the ejecta would be very small, resulting later in a He-dominated spectrum and a SN Ibn. In the case of the $25 M_{\odot}$ rotating model, there is no noticeable amount of $\mathrm{H}$ in its ejecta. As a consequence, any giant outburst that happens at the pre-SN stage of this model would produce a He-rich CSM and an ensuing type Ibn SN, similar to SN 2006jc (Pastorello et al. 2007).

Regarding the fate of the most massive stars $\left(M_{\text {ini }}>25 M_{\odot}\right)$, we reinforce that our models of single stars predict that they would evolve to the WR phase. Since this is at odds with the observational suggestion that LBVs with $M_{\text {ini }} \simeq 50 M_{\odot}$ are the progenitors of type IIn $\mathrm{SNe}$, let us examine the observational evidence of very massive LBVs exploding as SNe. Most of these come from the inference of large amounts of circumstellar material in the close vicinity of the progenitor star, such as in SN 2006gy (Smith et al. 2007).

In a few cases, a very luminous progenitor $\left(M_{V} \sim-10 \mathrm{mag}\right)$ has been detected in pre-explosion images, such as the progenitors of SN 2005gl (Gal-Yam \& Leonard 2009), SN 2009ip (Smith et al. 2010; Mauerhan et al. 2013; Foley et al. 2011; Pastorello et al. 2013), and SN 2010jl (Smith et al. 2011b). The high luminosity of the progenitor has been used as evidence for the star having initial masses $M_{\text {ini }}>50 M_{\odot}$. In the case of the progenitors of SN 2009ip, the $M_{V} \sim-10$ mag measurement seems to correspond to a phase when the star was quiescent (Smith et al. 2010). Thus, in this case, there is little doubt that the progenitor has $M_{\text {ini }}>50 M_{\odot}$, although one may wonder if the 2012 events observed in SN 2009ip correspond indeed to core collapse (Fraser et al. 2013). For the progenitors of SN 2005gl, however, there is no information about its preexplosion variability. One may wonder if the measured magnitude in the pre-explosion image (Gal-Yam \& Leonard 2009) was obtained during a giant outburst phase. During these giant outbursts, $M_{V}$ increases by 3 mag or more (Humphreys \& Davidson 1994) and, in this case, the progenitor of SN 2005gl could have had a much lower $M_{V} \sim-7$ mag during quiescence. This would imply an initial mass around $20-25 M_{\odot}$ (Fig. 6), which is consistent with our theoretical models of stars that explode during the LBV phase.

We see thus that observational evidences for luminous LBVs as $\mathrm{SN}$ progenitors is mounting, but one may wonder whether this is the most usual evolutionary path of massive stars or a peculiar case of massive star evolution, perhaps resulting from binary evolution (Vanbeveren et al. 2013). There is no doubt that some progenitors of core-collapse $\mathrm{SNe}$ have their structure resulting from interactions in close binaries and these cannot of course be described by our single-star models. If indeed high luminous LBV star explode as SNe, then this type of SNe would be difficult to explain in the frame of the single star scenario, as can be seen in Fig. 6. To obtain a clearer picture, a larger sample is needed, which may become possible with the current and next generation of transient surveys.

\section{Impacts on massive star evolution and concluding remarks}

We showed in this paper that producing detailed spectra out of evolutionary tracks significantly impacts the current paradigm of stellar evolution and how different spectral types are linked to the final evolutionary stages. Here we focused on the pre-SN stage of single stars. Based on a stellar evolution perspective, we will present in a forthcoming paper the results of the spectral classification of the evolutionary model outputs throughout their full lifetime. Nevertheless, we are already able to assess important aspects of massive star evolution and fate, as we summarize below.

1. The current rotating models from our group (Ekström et al. 2012) reproduce reasonably well the rates of different types of core-collapse SNe observed by S11 and E13, with the exception of the SN IIP rate, which is overestimated. The rates of $\mathrm{SN} \mathrm{Ib}$ and Ic predicted by the rotating models seem to be in line with the observations, although we note that the model rates may be overestimated, since we assume that all massive stars end their lives in a core-collapse SN event.

2. We performed combined stellar evolution and atmospheric modeling of massive stars at their pre-SN stage. With this approach, we are able to compute the emerging spectrum in high spectral resolution and perform synthetic photometry to obtain absolute magnitudes and bolometric corrections of the progenitors that have $T_{\mathrm{eff}}>8000 \mathrm{~K}$. For the remaining progenitors, we supplement our analysis by using public MARCS models, scaled to the luminosity and interpolated in $T_{\text {eff }}$ according to the end position in the HR diagram.

3. We found that massive stars, depending on their initial mass and rotation, end their lives as RSG, YHG, LBV, WN, or WO stars. For rotating models, we obtained the following types of SN progenitors: WO1-3 $\left(M_{\text {ini }} \geq 32 M_{\odot}\right)$, WN10-11 $\left(25<M_{\text {ini }}<32 M_{\odot}\right)$, LBV $\left(20 \leq M_{\text {ini }} \leq 25 M_{\odot}\right), \mathrm{G} 1 ~ I a^{+}$ $\left(18<M_{\text {ini }}<20 M_{\odot}\right)$, and RSGs $\left(9 \leq M_{\text {ini }} \leq 18 M_{\odot}\right)$. For non-rotating models, we found the following spectral types of the SN progenitors: WO1-3 $\left(M_{\text {ini }}>40 M_{\odot}\right)$, WN7-8 $\left(25<M_{\text {ini }} \leq 40 M_{\odot}\right), \mathrm{WN} 11 \mathrm{~h} / \mathrm{LBV}\left(20<M_{\text {ini }} \leq 25 M_{\odot}\right)$, and RSGs $\left(9 \leq M_{\text {ini }} \leq 20 M_{\odot}\right)$.

4. The most massive stars (initial mass above $32 M_{\odot}$ ) end their lives with extremely high $T_{\text {eff }}(150000-175000 \mathrm{~K})$. Their spectrum is characterized by broad emission lines of C IV and O VI, which are characteristic of WO stars. This is contrary to what has been widely expected based on the chemical abundance of the progenitor, which predicted a WC star at the pre-SN stage. Here we show that the high $T_{\text {eff }}$, coupled with the presence of moderate amounts of $\mathrm{O}$ at the surface ( $\sim 30 \%$ by mass), produces the morphology characteristic of WO 1-3 stars. For some WO models, we obtained spectral lines which are much weaker than observed in Galactic WOs, which may imply a hidden population of weak-lined WO stars or that our assumed values of $\dot{M}$ are too low. An increase in $\dot{M}$ by a factor of two is enough for producing a WO with an emission line strength in line with the observations.

5. We computed absolute magnitudes and bolometric corrections of core-collapse SN progenitors with initial mass in the range 9 to $120 M_{\odot}$. The behavior of the absolute magnitudes and bolometric corrections as a function of initial mass is regulated by how much flux from the star falls within the passband of a given filter. This depends on $T_{\text {eff }}, L_{\star}, \dot{M}$, and $v_{\infty}$. Therefore, not necessarily the most massive and luminous stars are the brighter ones in a given filter. We find that the RSGs are bright in the $R I J H K_{\mathrm{S}}$ filters and faint in 
the $F 170 W$ and $U B$ filters. LBVs, YHGs, and WNLs are relatively bright and WOs are faint in all optical/IR filters, with the exception of the $F 170 \mathrm{~W}$ filter.

6. We obtained a relationship between the absolute magnitude of RSGs in different filters and their initial mass. This can be used to estimate the initial mass of progenitors detected in the pre-explosion images of SN IIP. Our method provides similar values of the initial mass of RSG as those found in the literature, given the uncertainties.

7. We discussed the detectability of $\mathrm{SN} \mathrm{Ib}$ and Ic progenitors and argue that the WR stars that characterize the pre-SN phase are undetectable in the available pre-explosion images with the current magnitude limits. This is consistent with the current non-detection of WRs as progenitors of $\mathrm{SNe}$ in the available pre-explosion images of Eldridge et al. (2013).

8. Unlike the SN IIP progenitors, we find that the absolute magnitude in a given filter of SN Ic progenitors do not depend strongly on the initial mass. As a consequence, in the event that a type Ic progenitor is detected, our models indicate that it will be challenging to constrain its initial mass based on photometry (or spectroscopy) of the progenitor.

9. We predict that the limiting magnitudes in the observations should be at least 2 mag fainter than the current best limit in the $B$ band to detect single star progenitors of SN Ic at distances of 10-20 Mpc. For a SN 2002ap-like detection limit $\left(m_{B}=26.0 \mathrm{mag}\right.$, Crockett et al. 2007), the maximum distance that a SN Ic progenitor would be detected is $\sim 5.5 \mathrm{Mpc}$. For typical detection limits $(m=24.5 \mathrm{mag})$ and low amounts of extinction ( $0.3 \mathrm{mag})$, our models suggest that SN Ic progenitors from single stars would be detected up to a maximum distance of $\sim 2.7 \mathrm{Mpc}$.

Acknowledgements. We thank our referee, Jean-Claude Bouret, for a detailed reading and comments on the manuscript. We also thank John Hillier for making CMFGEN available, continuous support, and sharing models, and Jesús MaízAppelániz for making CHORIZOS available. We appreciate discussions with and comments on the manuscript from Simon Clark, Ben Davies, Luc Dessart, John Eldridge, John Hillier, Raphael Hirschi, Andreas Sander, and Jorick Vink. J.H.G. is supported by an Ambizione Fellowship of the Swiss National Science Foundation. C.G. acknowledges support from EU- FP7-ERC-2012-St Grant 306901.

\section{References}

Asplund, M., Grevesse, N., Sauval, A. J., \& Scott, P. 2009, ARA\&A, 47, 481 Barniske, A., Oskinova, L. M., \& Hamann, W.-R. 2008, A\&A, 486, 971 Boissier, S., \& Prantzos, N. 2009, A\&A, 503, 137

Cano, Z. 2013, MNRAS, 434, 1098

Clark, J. S., Larionov, V. M., \& Arkharov, A. 2005, A\&A, 435, 239

Clark, J. S., Crowther, P. A., Larionov, V. M., et al. 2009a, A\&A, 507, 1555

Clark, J. S., Davies, B., Najarro, F., et al. 2009b, A\&A, 504, 429

Clark, J. S., Castro, N., Garcia, M., et al. 2012a, A\&A, 541, A146

Clark, J. S., Najarro, F., Negueruela, I., et al. 2012b, A\&A, 541, A145

Clocchiatti, A. 2002, IAU Circ., 7793, 2

Cohen, M., Wheaton, W. A., \& Megeath, S. T. 2003, AJ, 126, 1090

Crockett, R. M., Smartt, S. J., Eldridge, J. J., et al. 2007, MNRAS, 381, 835

Crowther, P. A. 2001, in The Influence of Binaries on Stellar Population Studies,

ed. D. Vanbeveren, Astrophys. Space Sci. Lib., 264, 215

Crowther, P. A., Hillier, D. J., \& Smith, L. J. 1995, A\&A, 293, 172

Crowther, P. A., De Marco, O., \& Barlow, M. J. 1998, MNRAS, 296, 367

Davies, B., Kudritzki, R.-P., Plez, B., et al. 2013, ApJ, 767, 3

de Jager, C. 1998, A\&A Rev., 8, 145

de Jager, C., Nieuwenhuijzen, H., \& van der Hucht, K. A. 1988, A\&AS, 72, 259

de Mink, S. E., Langer, N., Izzard, R. G., Sana, H., \& de Koter, A. 2013, ApJ, 764,166

Dessart, L., Hillier, D. J., Livne, E., et al. 2011, MNRAS, 414, 2985

Dougherty, S. M., Clark, J. S., Negueruela, I., Johnson, T., \& Chapman, J. M. 2010, A\&A, 511, A58

Drew, J. E., Barlow, M. J., Unruh, Y. C., et al. 2004, MNRAS, 351, 206

Driebe, T., Groh, J. H., Hofmann, K., et al. 2009, A\&A, 507, 301
Drout, M. R., Soderberg, A. M., Gal-Yam, A., et al. 2011, ApJ, 741, 97 Egan, M. P., Clark, J. S., Mizuno, D. R., et al. 2002, ApJ, 572, 288 Ekström, S., Georgy, C., Eggenberger, P., et al. 2012, A\&A, 537, A146

Eldridge, J. J., Mattila, S., \& Smartt, S. J. 2007, MNRAS, 376, L52

Eldridge, J. J., Izzard, R. G., \& Tout, C. A. 2008, MNRAS, 384, 1109

Eldridge, J. J., Fraser, M., Smartt, S. J., Maund, J. R., \& Crockett, R. M. 2013, MNRAS, submitted [arXiv: 1301.1975]

Elias-Rosa, N., Van Dyk, S. D., Li, W., et al. 2009, ApJ, 706, 1174

Elias-Rosa, N., Van Dyk, S. D., Li, W., et al. 2010, ApJ, 714, L254

Elias-Rosa, N., Van Dyk, S. D., Li, W., et al. 2011, ApJ, 742, 6

Filippenko, A. V. 1997, ARA\&A, 35, 309

Filippenko, A. V., \& Chornock, R. 2002, IAU Circ., 7988, 2

Filippenko, A. V., \& Chornock, R. 2003, IAU Circ., 8084, 2

Foley, R. J., Berger, E., Fox, O., et al. 2011, ApJ, 732, 32

Fraser, M., Takáts, K., Pastorello, A., et al. 2010, ApJ, 714, L280

Fraser, M., Ergon, M., Eldridge, J. J., et al. 2011, MNRAS, 417, 1417

Fraser, M., Maund, J. R., Smartt, S. J., et al. 2012, ApJ, 759, L13

Fraser, M., Kotak, R., Pastorello, A., et al. 2013, The Astronomer's Telegram, 4953, 1

Gal-Yam, A., \& Leonard, D. C. 2009, Nature, 458, 865

Georgy, C. 2012, A\&A, 538, L8

Georgy, C., Meynet, G., Walder, R., Folini, D., \& Maeder, A. 2009, A\&A, 502, 611

Georgy, C., Ekström, S., Meynet, G., et al. 2012, A\&A, 542, A29

Giannone, P. 1967, ZAp, 65, 226

Gräfener, G., \& Hamann, W.-R. 2008, A\&A, 482, 945

Gräfener, G., Owocki, S. P., \& Vink, J. S. 2012, A\&A, 538, A40

Groh, J. H., \& Vink, J. S. 2011, A\&A, 531, L10

Groh, J. H., Damineli, A., Hillier, D. J., et al. 2009a, ApJ, 705, L25

Groh, J. H., Hillier, D. J., Damineli, A., et al. 2009b, ApJ, 698, 1698

Groh, J. H., Hillier, D. J., \& Damineli, A. 2011, ApJ, 736, 46

Groh, J. H., Hillier, D. J., Madura, T. I., \& Weigelt, G. 2012, MNRAS, 423, 1623

Groh, J. H., Meynet, G., \& Ekström, S. 2013, A\&A, 550, L7

Gustafsson, B., Edvardsson, B., Eriksson, K., et al. 2008, A\&A, 486, 951

Hamann, W.-R., Koesterke, L., \& Wessolowski, U. 1995, A\&AS, 113, 459

Hamann, W.-R., Gräfener, G., \& Liermann, A. 2006, A\&A, 457, 1015

Hamuy, M., Phillips, M., \& Thomas-Osip, J. 2003, IAU Circ., 8088, 3

Heger, A., Fryer, C. L., Woosley, S. E., Langer, N., \& Hartmann, D. H. 2003, ApJ, 591, 288

Hillier, D. J. 1987, ApJS, 63, 947

Hillier, D. J., \& Miller, D. L. 1998, ApJ, 496, 407

Hillier, D. J., Crowther, P. A., Najarro, F., \& Fullerton, A. W. 1998, A\&A, 340, 483

Hillier, D. J., Davidson, K., Ishibashi, K., \& Gull, T. 2001, ApJ, 553, 837

Hirschi, R., Meynet, G., \& Maeder, A. 2005, A\&A, 443, 581

Holberg, J. B., \& Bergeron, P. 2006, AJ, 132, 1221

Humphreys, R. M., \& Davidson, K. 1994, PASP, 106, 1025

Humphreys, R. M., \& McElroy, D. B. 1984, ApJ, 284, 565

Humphreys, R. M., Lamers, H. J. G. L. M., Hoekzema, N., \& Cassatella, A. 1989, A\&A, 218, L17

Kochanek, C. S., Khan, R., \& Dai, X. 2012, ApJ, 759, 20

Kotak, R., \& Vink, J. S. 2006, A\&A, 460, L5

Kovtyukh, V. V. 2007, MNRAS, 378, 617

Kudritzki, R.-P., \& Puls, J. 2000, ARA\&A, 38, 613

Lamers, H. J. G. L. M., Snow, T. P., \& Lindholm, D. M. 1995, ApJ, 455, 269

Langer, N. 2012, ARA\&A, 50, 107

Langer, N., Norman, C. A., de Koter, A., et al. 2007, A\&A, 475, L19

Leitherer, C., Allen, R., Altner, B., et al. 1994, ApJ, 428, 292

Levesque, E. M., Massey, P., Olsen, K. A. G., et al. 2005, ApJ, 628, 973

Levesque, E. M., Kewley, L. J., Berger, E., \& Zahid, H. J. 2010, AJ, 140, 1557

Li, W., Van Dyk, S. D., Filippenko, A. V., et al. 2006, ApJ, 641, 1060

Li, W., Wang, X., Van Dyk, S. D., et al. 2007, ApJ, 661, 1013

Lobel, A., Dupree, A. K., Stefanik, R. P., et al. 2003, ApJ, 583, 923

Maeder, A. 1997, A\&A, 321, 134

Maeder, A., \& Meynet, G. 2000, ARA\&A, 38, 143

Maeder, A., \& Meynet, G. 2012, Rev. Mod. Phys., 84, 25

Maíz-Apellániz, J. 2004, PASP, 116, 859

Maíz Apellániz, J. 2006, AJ, 131, 1184

Maíz-Apellániz, J. 2007, in The Future of Photometric, Spectrophotometric and Polarimetric Standardization, ed. C. Sterken, ASP Conf. Ser., 364, 227

Marcolino, W. L. F., de Araújo, F. X., Lorenz-Martins, S., \& Fernandes, M. B. 2007, AJ, 133, 489

Mauerhan, J. C., Smith, N., Filippenko, A., et al. 2013, MNRAS, 430, 1801

Maund, J. R., \& Smartt, S. J. 2005, MNRAS, 360, 288

Maund, J. R., Smartt, S. J., \& Danziger, I. J. 2005a, MNRAS, 364, L33

Maund, J. R., Smartt, S. J., \& Schweizer, F. 2005b, ApJ, 630, L33

Maund, J. R., Fraser, M., Ergon, M., et al. 2011, ApJ, 739, L37 
Maund, J., Reilly, E., \& Mattila, S. 2013, MNRAS, submitted [arXiv: 1302 . 7152]

Meynet, G., \& Maeder, A. 2003, A\&A, 404, 975

Meynet, G., Haemmerlé, L., Ekström, S., et al. 2013, in EAS Pub. Ser. 60, eds. P. Kervella, T. Le Bertre, \& G. Perrin, 17

Moriya, T. J., Groh, J. H., \& Meynet, G. 2013, A\&A, 557, L2

Najarro, F. 2001, in P Cygni 2000: 400 Years of Progress, eds. M. de Groot, \& C. Sterken, ASP Conf. Ser., 233, 133

Najarro, F., Hillier, D. J., \& Stahl, O. 1997, A\&A, 326, 1117

Najarro, F., Figer, D. F., Hillier, D. J., Geballe, T. R., \& Kudritzki, R. P. 2009, ApJ, 691, 1816

Nugis, T., \& Lamers, H. J. G. L. M. 2000, A\&A, 360, 227

Pastorello, A., Smartt, S. J., Mattila, S., et al. 2007, Nature, 447, 829

Pastorello, A., Cappellaro, E., Inserra, C., et al. 2013, ApJ, 767, 1

Pauldrach, A. W. A., \& Puls, J. 1990, A\&A, 237, 409

Phillips, M. M., Suntzeff, N. B., Krisciunas, K., et al. 2001, IAU Circ., 7772, 2

Podsiadlowski, P., Joss, P. C., \& Hsu, J. J. L. 1992, ApJ, 391, 246

Reimers, D. 1975, Mem. Soc. Roy. Sci. Liege, 8, 369

Reimers, D. 1977, A\&A, 61, 217

Ritchie, B. W., Clark, J. S., Negueruela, I., \& Najarro, F. 2009, A\&A, 507, 1597

Salasnich, B., Bressan, A., \& Chiosi, C. 1999, A\&A, 342, 131

Sana, H., de Mink, S. E., de Koter, A., et al. 2012, Science, 337, 444

Sander, A., Hamann, W.-R., \& Todt, H. 2012, A\&A, 540, A144

Smartt, S. J. 2009, ARA\&A, 47, 63

Smartt, S. J., Gilmore, G. F., Trentham, N., Tout, C. A., \& Frayn, C. M. 2001, ApJ, 556, L29

Smartt, S. J., Maund, J. R., Gilmore, G. F., et al. 2003, MNRAS, 343, 735

Smartt, S. J., Eldridge, J. J., Crockett, R. M., \& Maund, J. R. 2009, MNRAS, 395,1409

Smith, L. F., \& Maeder, A. 1991, A\&A, 241, 77

Smith, L. J., Crowther, P. A., \& Prinja, R. K. 1994, A\&A, 281, 833

Smith, L. F., Shara, M. M., \& Moffat, A. F. J. 1996, MNRAS, 281, 163
Smith, N., Vink, J. S., \& de Koter, A. 2004, ApJ, 615, 475 Smith, N., Li, W., Foley, R. J., et al. 2007, ApJ, 666, 1116 Smith, N., Miller, A., Li, W., et al. 2010, AJ, 139, 1451

Smith, N., Li, W., Filippenko, A. V., \& Chornock, R. 2011a, MNRAS, 412, 1522 Smith, N., Li, W., Miller, A. A., et al. 2011b, ApJ, 732, 63

Stahl, O., Jankovics, I., Kovács, J., et al. 2001, A\&A, 375, 54

Sylvester, R. J., Skinner, C. J., \& Barlow, M. J. 1998, MNRAS, 301, 1083

Trams, N. R., van Tuyll, C. I., Voors, R. H. M., et al. 1999, in IAU Colloq.

169: Variable and Non-spherical Stellar Winds in Luminous Hot Stars, eds. B. Wolf, O. Stahl, \& A. W. Fullerton (Berlin: Springer Verlag), Lect. Notes Phys., 523, 71

Van Dyk, S. D., Li, W., \& Filippenko, A. V. 2003, PASP, 115, 448

Van Dyk, S. D., Cenko, S. B., Poznanski, D., et al. 2012, ApJ, 756, 131

van Genderen, A. M. 2001, A\&A, 366, 508

van Loon, J. T., Groenewegen, M. A. T., de Koter, A., et al. 1999, A\&A, 351, 559

van Loon, J. T., Cioni, M.-R. L., Zijlstra, A. A., \& Loup, C. 2005, A\&A, 438, 273

Vanbeveren, D., De Loore, C., \& Van Rensbergen, W. 1998, A\&ARv, 9, 63

Vanbeveren, D., Mennekens, N., Van Rensbergen, W., \& De Loore, C. 2013, A\&A, 552, A105

Vink, J. S. 2012, in Astrophys. Space Sci. Lib., eds. K. Davidson, \& R. M. Humphreys, 384, 221

Vink, J. S., \& de Koter, A. 2002, A\&A, 393, 543

Vink, J. S., de Koter, A., \& Lamers, H. J. G. L. M. 1999, A\&A, 350, 181

Vink, J. S., de Koter, A., \& Lamers, H. J. G. L. M. 2001, A\&A, 369, 574

Walborn, N. R., \& Fitzpatrick, E. L. 2000, PASP, 112, 50

Walmswell, J. J., \& Eldridge, J. J. 2012, MNRAS, 419, 2054

Yoon, S.-C., \& Cantiello, M. 2010, ApJ, 717, L62

Yoon, S.-C., Gräfener, G., Vink, J. S., Kozyreva, A., \& Izzard, R. G. 2012, A\&A 544, L11

Zahn, J.-P. 1992, A\&A, 265, 115 